\title{
HITTING DISTRIBUTION TO A QUADRANT OF TWO-DIMENSIONAL RANDOM WALK
}

\author{
YASUNARI FUKAI
}

Let $H_{L}(\xi, \eta)$ be the probability that a two-dimensional simple random walk starting at $\xi$ hits the third quadrant $L$ for the first time at $\eta$. The main objective of this paper is to investigate the asymptotic behavior of $H_{L}(\xi, \eta)$. It is especially proved that there exists a constant $C_{0}$ such that for $\xi \in Z^{2} \backslash L$ and $l \in N$,

$$
\left|H_{L}(\xi,(-l, 0))-h_{L}(\xi,(-l, 0))\right| \leq C_{0}\left\{|\xi+(l, 0)|^{-3}+|\xi|^{-2 / 3} l^{-5 / 3}\right\},
$$

where $h_{L}(\xi, \cdot)$ is the density of the hitting distribution to the third quadrant of two-dimensional standard Brownian motion starting at $\xi$. This estimate is sharp at least in the sense that the powers $-2 / 3$ and $-5 / 3$ can not be improved.

\section{Introduction and statements of results}

Let $\{S(n)\}_{n=0}^{\infty}$ be a two-dimensional simple random walk starting at $\xi \in Z^{2}$, namely,

$$
S(0)=\xi \quad \text { and } \quad S(n)=S(0)+\sum_{k=1}^{n} X_{k},
$$

where $X_{1}, X_{2}, \ldots$ is a sequence of independent, identically distributed random variables that take four values $(1,0),(-1,0),(0,1),(0,-1)$ with equal probability. We denote by $P_{\xi}$ the probability law of the process $\{S(n)\}_{n=0}^{\infty}$. For a subset $A$ of $\boldsymbol{R}^{2}$ such that $A \cap \boldsymbol{Z}^{2} \neq \emptyset$, define

$$
\tau_{A}=\inf \{n \geq 1: S(n) \in A\},
$$

the hitting time of $A$. Since $S(n)$ is recurrent, $\tau_{A}<\infty$ a.s. The hitting distribution $H_{A}(\xi, \eta)$ is defined by

$$
H_{A}(\xi, \eta)=P_{\xi}\left\{S\left(\tau_{A}\right)=\eta\right\}, \quad\left(\xi \in Z^{2} \backslash A, \eta \in A \cap Z^{2}\right) .
$$

1991 Mathematics Subject Classifications: Prımary 60J15; secondary 60J45.

Keywords and phrases: Two-dimensional simple random walk, hittıng distribution, twodimensional standard Brownian motion, Green's function.

Received December 17, 1998; revised July 29, 1999. 
By the definition of simple random walk, this is a probability measure on

$$
\partial\left(\boldsymbol{Z}^{2} \backslash A\right):=\left\{\eta \in \boldsymbol{Z}^{2} \cap A\left|\exists \zeta \in \boldsymbol{Z}^{2} \backslash A:\right| \eta-\zeta \mid=1\right\} .
$$

We use complex notation $\zeta=\zeta_{1}+i \zeta_{2} \in C, i=\sqrt{-1}$, instead of $\zeta=\left(\zeta_{1}, \zeta_{2}\right) \in \boldsymbol{R}^{2}$ for the sake of convenience: The notations $|\zeta|, \bar{\zeta}$ and $\arg (\xi)$ are used in the usual sense. Put

$$
L=\{\zeta \in C: \mathfrak{R}(\zeta) \leq 0 \text { and } \mathfrak{I}(\zeta) \leq 0\}
$$

(the third quadrant of $C$ ) and

$$
\boldsymbol{C}^{*}=\{\zeta \in \boldsymbol{C}: \mathfrak{R}(\zeta), \mathfrak{I}(\zeta) \in \boldsymbol{Z}\}
$$

(the set of all lattice points of $C$ ). Let $W(t)$ be a two-dimensional standard Brownian motion on a probability space $(\Omega, \mathscr{F}, \mathscr{P})$ and $T_{A}$ the corresponding hitting time:

$$
T_{A}=\inf \{t \geq 0: W(t) \in A\} .
$$

Let $h_{A}$ denote the Brownian analogue of $H_{A}$, i.e., for $x+i y \in C \backslash L, z>0$,

$$
h_{L}(x+i y,-z)=\frac{\partial}{\partial z} \mathscr{P}\left\{-z<W\left(T_{L}\right) \leq 0 \mid W(0)=x+i y\right\} .
$$

The objective of this paper is to investigate the asymptotic behavior of $H_{L}$. The function $h_{L}$ has an explicit expression by means of a Bessel function, so that fine bounds for the difference $H_{L}-h_{L}$ will provide fine estimates of $H_{L}$. The main results are stated as Theorems 1.1 through 1.8 below.

THEOREM 1.1. There exists a constant $C_{0}$ such that for $\xi \in C^{*} \backslash L$ and $l \in N$,

$$
\left|H_{L}(\xi,-l)-h_{L}(\xi,-l)\right| \leq C_{0}\left\{|\xi+l|^{-3}+|\xi|^{-2 / 3} l^{-5 / 3}\right\} .
$$

THEOREM 1.2. For any $\xi \in C^{*} \backslash L$, there exists a positive constant $c_{1}(\xi)$ such that for $l \in N$,

$$
H_{L}(\xi,-l) \geq c_{1}(\xi) l^{-5 / 3}
$$

THEOREM 1.3. For $\delta \in(0, \pi / 2)$ and $l \in N$, there exists a positive constant $\hat{c}_{1}(\delta, l)$ such that for $\xi \in C^{*} \backslash L$ with $-(\pi / 2)+\delta<\arg (\xi)<\pi-\delta$,

$$
H_{L}(\xi,-l) \geq \hat{c}_{1}(\delta, l)|\xi|^{-2 / 3} .
$$

Remark 1.1. For $x, z \in(0,+\infty)$, we can write

$$
h_{L}(x,-z)=\frac{2}{3 \pi}\left(\frac{x}{x^{2}+z^{2}}\right)^{2 / 3}\left(\frac{1}{z}\right)^{1 / 3}\left(\sin \frac{\pi}{3}\right) M(x, z),
$$

where $M(x, z)$ is bounded both from above and from below by positive constants and satisfies 


$$
M(x, z) \rightarrow 1 \text { as }(x / z) \vee(z / x) \rightarrow+\infty
$$

(see (2.12) and (2.13) below), where $a \vee b=\max \{a, b\}$. From this, the estimate of $H_{L}(k,-l)$ in Theorem 1.1 is seen to be quite sharp when both $k$ and $l$ are large (in fact the powers $-2 / 3$ and $-5 / 3$ appearing in (1.2) cannot be improved; see Remark 1.4). However it does not give the lower bounds of $H_{L}$ as Theorem 1.2 and Theorem 1.3 do.

Corollary 1.4. For any $\delta \in(0, \pi / 2)$, there exists a positive constant $c$ such that for $l \in N$ and $\xi \in C^{*} \backslash L$ with $-(\pi / 2)+\delta<\arg (\xi)<\pi-\delta$,

$$
\frac{1}{c} h_{L}(\xi,-l) \leq H_{L}(\xi,-l) \leq c h_{L}(\xi,-l) .
$$

By means of Green's function

$$
G_{A}(\xi, \eta):=\sum_{n=0}^{\infty} P_{\xi}\left\{S(n)=\eta, \tau_{A}>n\right\},
$$

$H_{A}(\xi, \eta)$ can be expressed as

$$
H_{A}(\xi, \eta)=\sum_{\zeta \in C^{*} \backslash A} G_{A}(\xi, \zeta) P_{\zeta}\{S(1)=\eta\} \quad\left(\xi \in C^{*} \backslash A, \eta \in A \cap C^{*}\right)
$$

(see Spitzer $[8, \S 15])$. Put

$$
B=\{\zeta \in C: \mathfrak{I}(\zeta) \leq 0\} .
$$

The reflection principle then shows that for $\xi, \eta \in C^{*} \backslash B$,

$$
G_{B}(\xi, \eta)=a(\xi-\bar{\eta})-a(\xi-\eta)
$$

where $a(\xi)$ is the potential function, that is,

$$
a(\xi):=\sum_{n=0}^{\infty}\left(P_{0}\{S(n)=0\}-P_{\xi}\{S(n)=0\}\right) .
$$

It is well known (Spitzer [8, p. 148]) that

$$
\begin{aligned}
a(0)=0, & \\
a(1)= & a(-1)=a(i)=a(-i)=1, \\
a(2)= & a(-2)=a(2 i)=a(-2 i)=4-\frac{8}{\pi}, \\
& a(k+i k)=\frac{4}{\pi} \sum_{j=1}^{k} \frac{1}{2 j-1} .
\end{aligned}
$$

Fukai and Uchiyama [2] provided the following asymptotic expansion for $a(\xi)$ : 


$$
a(\xi)=\frac{2}{\pi} \log |\xi|+\frac{\log 8+2 \gamma}{\pi}+\frac{1}{6 \pi} \cdot \frac{8\left(\omega_{1}^{\xi} \omega_{2}^{\xi}\right)^{2}-1}{|\xi|^{2}}+O\left(|\xi|^{-4}\right)
$$

where $\omega^{\xi}=\left(\omega_{1}^{\xi}, \omega_{2}^{\xi}\right)=\xi /|\xi|$ and $\gamma$ is Euler's constant, and by using this, the estimate

$$
H_{B}(i k, l)=h_{B}(i k, l)+O\left(|i k+l|^{-3}\right),
$$

where $h_{B}=h_{B}(x+i y, z)$ is a Brownian analogue of $H_{B}$ and explicitly given by

$$
h_{B}(x+i y, z)=\frac{1}{\pi} \frac{y}{y^{2}+(z-x)^{2}} .
$$

The estimate (1.7) will play a crucial role in the proof of Theorem 1.1.

Remark 1.2. Let

$$
\begin{aligned}
B^{\prime} & =\{\zeta \in C:-\mathfrak{R}(\zeta) \geq \mathfrak{I}(\zeta)\}, \\
V & =\{\zeta \in C:-\mathfrak{R}(\zeta) \leq \mathfrak{I}(\zeta)\} \cup\{\zeta \in C: \mathfrak{R}(\zeta) \geq \mathfrak{I}(\zeta)\}
\end{aligned}
$$

(see Figure 1) then the reflection principle gives the relations

$$
G_{B^{\prime}}(\xi, \eta)=a(\xi+i \bar{\eta})-a(\xi-\eta), \quad\left(\xi, \eta \in C^{*} \backslash B^{\prime}\right)
$$

and

$$
G_{V}(\xi, \eta)=a(-\xi-i \bar{\eta})-a(-\xi+\eta)+a(-\xi+i \bar{\eta})-a(-\xi-\eta), \quad\left(\xi, \eta \in C^{*} \backslash V\right) .
$$

We can also compute the following estimates. For $\xi \in C^{*} \backslash B^{\prime}$ and $l \in Z$,

$$
\begin{aligned}
H_{B^{\prime}}(\xi, l-i l)= & h_{B^{\prime}}(\xi, l-i l)+\frac{1}{2 \pi} \frac{\xi_{1}+\xi_{2}}{|\xi-l+i l|^{4}} \\
& -\frac{4}{\pi} \frac{\left(\xi_{1}-l\right)^{3}+\left(\xi_{2}+l\right)^{3}}{|\xi-l+i l|^{6}}+\frac{4}{\pi} \frac{\left(\xi_{1}-l\right)^{5}+\left(\xi_{2}+l\right)^{5}}{|\xi-l+i l|^{8}} \\
& +O\left(|\xi-l+i l|^{-4}\right),
\end{aligned}
$$

where
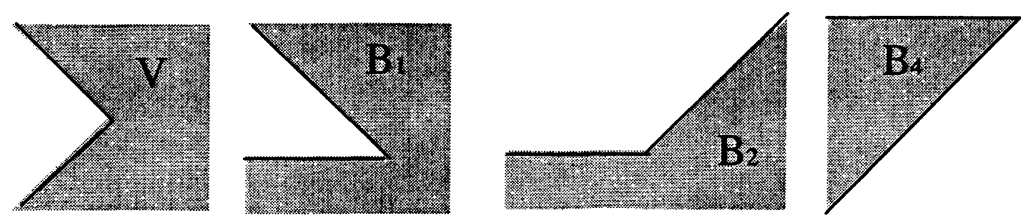

Figure 1: The regions $V, B_{1}, B_{2}$ and $B_{4}$. 


$$
h_{B^{\prime}}(x+i y, z-i z)=\frac{1}{\pi} \frac{x+y}{(x-z)^{2}+(y+z)^{2}} .
$$

For $\xi \in C^{*} \backslash V$ and $l \in N$,

$$
H_{V}(\xi,-l+i l)=h_{V}(\xi,-l+i l)+O\left(|\xi+l-i l|^{-3}\right),
$$

where $h_{V}(x+i y,-z+i z)=h_{B^{\prime}}(-x-i y, z-i z)-h_{B^{\prime}}(-x-i y,-z+i z)$.

There is, however, no simple relation between $G_{L}$ and $a(\xi)$ as those for $G_{B}$, $G_{B^{\prime}}$ and $G_{V}$. To calculate $H_{L}$, we derive relations among $H_{L}, h_{L}, H_{B}$ and $h_{B}$. By using (1.7) and an explicit representation of $h_{L}$ as given later, such relations lead to the estimate of Theorem 1.1.

Let

$$
\begin{aligned}
& B_{2}=\{\zeta \in C: \mathfrak{I}(\zeta) \leq 0\} \cup\{\zeta \in C: \mathfrak{R}(\zeta) \geq \mathfrak{I}(\zeta)\} \\
& B_{4}=\{\zeta \in C: \mathfrak{R}(\zeta) \leq \mathfrak{I}(\zeta) \leq 0\}
\end{aligned}
$$

(see Figure 1). The same method as proving Theorem 1.1 can be adapted for deriving (6.6) in $\$ 6$ and the following estimates. There exists a constant $C$ such that for $\xi \in C^{*} \backslash B_{2}$ and $\eta \in \partial\left(C^{*} \backslash B_{2}\right)$,

$$
\left|H_{B_{2}}(\xi, \eta)-h_{B_{2}}(\xi, \eta)\right| \leq C\left\{|\xi-\eta|^{-3}+|\xi|^{-2 / 3}(|\eta|+1)^{-5 / 3}\left(|\xi|^{-2 / 3} \wedge(|\eta|+1)^{-2 / 3}\right)\right\},
$$

and for $\xi \in C^{*} \backslash B_{4}$ and $\eta \in \partial\left(C^{*} \backslash B_{4}\right)$,

$$
\left|H_{B_{4}}(\xi, \eta)-h_{B_{4}}(\xi, \eta)\right| \leq C\left\{|\xi-\eta|^{-3}+|\xi|^{-4 / 7}(|\eta|+1)^{-11 / 7}\right\}
$$

where $h_{A}(x+i y, \cdot)$ is the density relative to Lebesgue measure on the boundary of $A$ of the hitting distribution to $A$ of two-dimensional standard Brownian motion starting at $x+i y$.

Put

$$
B_{1}=\{\zeta \in C:-\mathfrak{R}(\zeta) \leq \mathfrak{I}(\zeta)\} \cup\{\zeta \in C: \mathfrak{I}(\zeta) \leq 0\}
$$

(see Figure 1). It is easy to see that for $\xi \in C^{*} \backslash B_{1}$ and $\eta \in \partial\left(C^{*} \backslash B_{1}\right)$,

$$
H_{B_{1}}(\xi, \eta)=h_{B_{1}}(\xi, \eta)+O\left(|\xi-\eta|^{-3}\right)
$$

from (1.7), (1.9) and the relations

$$
\begin{aligned}
H_{B_{1}}(\xi,-l+i l)= & H_{B^{\prime}}(-\xi, l-i l)-H_{B^{\prime}}(-\xi,-l+i l) \\
& -H_{B^{\prime}}(-\bar{\xi}, l-i l)+H_{B^{\prime}}(-\bar{\xi},-l+i l), \\
H_{B_{1}}(\xi,-l)= & H_{B}(\xi,-l)-H_{B}(\xi, l)-H_{B}(-i \xi, l)+H_{B}(-i \xi,-l),
\end{aligned}
$$

that are given by the reflection principle and symmetry of simple random walk.

The estimates (1.10) and (1.11) are sharp at least in the sense that $O\left(|\xi-\eta|^{-3}\right)$ can not be replaced by $o\left(|\xi-\eta|^{-3}\right)$. For $s \in(0,1)$ and $l>1 / s$, we put $\xi_{l}^{s}=-l+i[s l]$, where $[a]$ denotes the greatest integer not greater than $a$. Then from (1.9) and (1.12) it is easy to deduce that there exist constants 
$s_{0} \in(0,1), l_{0} \in \boldsymbol{N}$ and $C>0$ such that for $l \geq l_{0}$,

$$
H_{B_{1}}\left(\xi_{l}^{s_{0}},-l+i l\right)-h_{B_{1}}\left(\xi_{l}^{s_{0}},-l+i l\right) \geq C\left(1-s_{0}\right)^{-3} l^{-3} .
$$

Thus $O\left(|\xi-\eta|^{-3}\right)$ cannot be replaced by $o\left(|\xi-\eta|^{-3}\right)$ in (1.11). The reasoning for $(1.10)$ is similar.

THEOREM 1.5. For any $k \in N$, the following limit exists

$$
c^{*}(k):=\lim _{l \rightarrow \infty} l^{5 / 3} H_{L}(k,-l) .
$$

The function $c^{*}$ is characterized as a unique solution to the equation

$$
c^{*}(k)=\sum_{j=1}^{\infty} H_{B}(i k, j) c^{*}(j), \quad(k \in N)
$$

with the boundary condition $\lim _{k \rightarrow \infty} k^{-2 / 3} c^{*}(k)=c_{0}$, where $c_{0}=(2 / 3 \pi) \sin (\pi / 3)$.

THEOREM 1.6. For any $l \in N$, the following limit exists

$$
c_{*}(l):=\lim _{k \rightarrow \infty} k^{2 / 3} H_{L}(k,-l) .
$$

The function $c_{*}$ is characterized as a unique solution to the equation

$$
c_{*}(l)=\sum_{j=1}^{\infty} c_{*}(j) H_{B}(i j, l), \quad(l \in \mathbf{N})
$$

with the boundary condition $\lim _{l \rightarrow \infty} l^{1 / 3} c_{*}(l)=c_{0}$, where $c_{0}$ is the same constant as in Theorem 1.5. Moreover, $\lim _{k \rightarrow \infty} k^{2 / 3} H_{L}(k, 0)$ exists and the limit is expressed by means of $c_{*}$ and $H_{B}$ as

$$
\lim _{k \rightarrow \infty} k^{2 / 3} H_{L}(k, 0)=2 \sum_{j=1}^{\infty} c_{*}(j) H_{B}(i j, 0) .
$$

Remark 1.3. We can show that for $\xi \in C^{*} \backslash L$, there exists $\lim _{l \rightarrow \infty} l^{5 / 3}$. $H_{L}(\xi,-l)$. The limit is expressed by means of $c^{*}$ and $H_{B}$ as follows:

$$
\lim _{l \rightarrow \infty} l^{5 / 3} H_{L}(\xi,-l)= \begin{cases}\sum_{j=1}^{\infty} H_{B}(\xi, j) c^{*}(j), & \mathfrak{R}(\xi) \leq \mathfrak{I}(\xi) \\ \sum_{j=1}^{\infty} H_{B}(i \bar{\xi}, j) c^{*}(j), & \mathfrak{R}(\xi)>\mathfrak{I}(\xi) .\end{cases}
$$

It is related to $\lambda_{1}(\xi, \pi, \pi / 2)$ appearing in Kesten [5, Theorem 1] as follows:

$$
\lim _{l \rightarrow \infty} l^{5 / 3} H_{L}(\xi,-l)=|\xi|^{2 / 3} \lambda_{1}(\xi, \pi, \pi / 2) \frac{2}{3 \pi} \sin \left(\frac{2 \theta+\pi}{3}\right),
$$


where $(|\xi|, \theta)$ is the polar coordinate of $\xi,-\pi / 2<\theta<\pi$. Notice that $\xi$ and $i \bar{\xi}$ are mirror images of each other with respect to the diagonal $\{x+i x: x \in \boldsymbol{R}\}$. The representation (1.16) gives

$$
\lim _{l \rightarrow \infty} l^{5 / 3} H_{L}(\xi,-l)=\lim _{l \rightarrow \infty} l^{5 / 3} H_{L}(i \bar{\xi},-l) .
$$

Since $\lim _{l \rightarrow \infty} l^{5 / 3} H_{L}(\xi,-l)$ is quite sensitive to the change of $\xi$ as realized e.g., by the fact that $\lim _{l \rightarrow \infty} l^{5 / 3} H_{L}(i,-l)$ is equal to

$$
\frac{1}{4}\left\{\lim _{l \rightarrow \infty} l^{5 / 3} H_{L}(2 i,-l)+\lim _{l \rightarrow \infty} l^{5 / 3} H_{L}(1+i,-l)+\lim _{l \rightarrow \infty} l^{5 / 3} H_{L}(-1+i,-l)\right\},
$$

(1.18) may seem strange coincidence. It, however, simply explained by

$$
\left|H_{L}(\xi,-l)-H_{L}(i \bar{\xi},-l)\right| \leq H_{B}(\xi,-l) \quad(\text { if } \mathfrak{R}(\xi) \leq \mathfrak{I}(\xi))
$$

(as is seen by reflection with respect to the diagonal $\mathfrak{R}(\xi)=\mathfrak{I}(\xi)$ ). Moreover, we can show that for $\theta \in[\pi / 4, \pi)$,

$$
\lim _{\substack{\arg (\xi) \rightarrow \theta \\|\xi| \rightarrow \infty}}|\xi|^{2 / 3} H_{L}(\xi,-l)=c_{*}(l) \frac{1}{\pi} \int_{0}^{\infty} \frac{\sin \theta}{\sin ^{2} \theta+(\cos \theta-u)^{2}} u^{-2 / 3} d u .
$$

In the particular case when $\xi=i k$ we have

$$
\lim _{k \rightarrow \infty} k^{2 / 3} H_{L}(i k,-l)=c_{*}(l) .
$$

Remark 1.4. We shall see in $\$ 4$ that neither of $k^{-2 / 3} c^{*}(k)$ and $l^{1 / 3} c_{*}(l)$ is constant, while both $x^{-2 / 3} \lim _{z \rightarrow \infty} z^{5 / 3} h_{L}(x,-z)$ and $z^{1 / 3} \lim _{x \rightarrow \infty} x^{2 / 3} h_{L}(x,-z)$ are constant $\left(=c_{0}\right)$. This is the reason that the powers $-2 / 3$ and $-5 / 3$ appearing (1.2) cannot be improved. By Theorem 1.1,

$$
c_{*}(l) l^{1 / 3}-c_{0}=O\left(l^{-4 / 3}\right),
$$

and for $\xi \in C^{*} \backslash L$,

$$
\left|\lim _{l \rightarrow \infty} l^{5 / 3} H_{L}(\xi,-l) / \lim _{l \rightarrow \infty} l^{5 / 3} h_{L}(\xi,-l)-1\right| \leq C_{1}|\xi|^{-4 / 3} \frac{1}{\sin \left(\left(2 \theta_{0}+\pi\right) / 3\right)},
$$

where $\left(|\xi|, \theta_{0}\right)$ is the polar coordinate of $\xi,-\pi / 2<\theta_{0}<\pi$ and $C_{1}$ is a constant independent of $\xi$; in particular $c^{*}(k) k^{-2 / 3}-c_{0}=O\left(k^{-4 / 3}\right)$.

Remark 1.5. With the help of invariance principle and a simple estimate of the difference $H_{L}(\xi,-l)-H_{L}(\xi,-l-1)$ (see Remark 2.1 in $\S 2$ ), we can readily deduce from Theorem 1 in Kesten [5] that there exists $\lim _{l \rightarrow \infty} l^{5 / 3} H_{L}(\xi,-l)$ and these limits are positive and satisfy (1.17). In Kesten [5], however, it does not seem to be identified with anything. We also deduce from Theorem 2 in Kesten [5] that for $\varepsilon \in(0,1 / 8]$, there exists a constant $K^{0}=K^{0}(\varepsilon)<\infty$ and a function $\theta(\varepsilon, l) \geq 0$ such that

$$
\theta(\varepsilon, l) \rightarrow 0 \text { as } l \rightarrow \infty
$$


and for each $\xi \in C^{*} \backslash L$ with $|\xi| \leq(1-2 \varepsilon) l$

$$
\begin{aligned}
\left|H_{L}(\xi,-l)-h_{L}(\xi,-l)\right| \leq & K^{0}\left\{|\xi|^{-1 / 3}+\frac{\rho(|\xi| / l)+(1 / l)}{(|\xi| / l)}\right\} l^{-5 / 3} \\
& +\theta(\varepsilon, l) \frac{\rho(|\xi| / l)+(1 / l)}{(|\xi| / l)}|\xi|^{2 / 3} l^{-5 / 3}
\end{aligned}
$$

where

$$
\rho(\xi)= \begin{cases}\inf \{|\xi-x|: x \leq 0\} & \text { if } \xi_{2} \geq 0 \\ \inf \{|\xi-i x|: x \leq 0\} & \text { if } \xi_{2}<0\end{cases}
$$

Theorem 1.1 is an improvement of this estimate.

For a finite subset $A$ of $C^{*}$, the hitting distribution of $A$ by the random walk starting at infinity may be defined by

$$
\mu_{A}(\zeta):=\lim _{|\xi| \rightarrow \infty} H_{A}(\xi, \zeta) \quad(\zeta \in A)
$$

(the limit exists and $\sum_{\zeta \in A} \mu_{A}(\zeta)=1$, Spitzer $\left.[8, \S 14]\right)$. Let

$$
L(n)=\left\{-l \in C^{*}: 0 \leq l \leq n\right\} \cup\left\{-i l \in C^{*}: 0 \leq l \leq n\right\} .
$$

Theorem 1.6 is useful for investigating the asymptotic behavior of $\mu_{L(n)}(-l)$.

THEOREM 1.7. There exists a positive constant $\alpha$ such that

$$
\lim _{n \rightarrow \infty} n^{2 / 3} \mu_{L(n)}(-l)=\alpha c_{*}(l), \quad(l \in N),
$$

where $c_{*}$ is the same function as in Theorem 1.6.

Remark 1.6. The existence of $\lim _{n \rightarrow \infty} n^{2 / 3} \mu_{L(n)}(-l)$ is obtained, as a special case, by Kesten [5], in which, however, the identification of it as in Theorem 1.7 is not considered.

Remark 1.7. Auer [1], Kesten [4] and Lawler [7, \$2.4] provided some bounds of the hitting probabilities $\mu_{A}$. In those papers, Auer, Kesten and Lawler used the fact that there is a positive constant $C$ such that if a finite connected set $A \subset C^{*}$ contains the origin and $\eta \in A$ then

$$
\frac{1}{C} P_{\eta}\left\{\tau_{\partial C(2 r)}<\tau_{A}\right\} \leq \mu_{A}(\eta) \leq C P_{\eta}\left\{\tau_{\partial C(2 r)}<\tau_{A}\right\}
$$

where $\quad r=r(A):=\max \{|\xi|: \xi \in A\}, C(r)=\left\{\xi \in C^{*}:|\xi| \leq r\right\} \quad$ and $\quad \partial C(r)=$ $\left\{\xi \in C^{*} \backslash C(r)|\exists \zeta \in C(r):| \xi-\zeta \mid=1\right\}$ (as in (1.1)) and computed $P_{\eta}\left\{\tau_{\partial C(2 r)}<\tau_{A}\right\}$ instead of $\mu_{A}(\eta)$. This method is an important tool to compute a bound of $\mu_{A}(\eta)$ but would give no information about $l^{1 / 3} \lim _{n \rightarrow \infty} n^{2 / 3} \mu_{L(n)}(-l)$ except for boundedness. 
THEOREM 1.8. Let $g_{L}$ be the Green function of $L$ for two-dimensional standard Brownian motion. Then there exists a constant $\bar{C}_{0}$ such that for $\xi, \eta \in$ $C^{*} \backslash L$ with $\xi \neq \eta$

$$
\left|G_{L}(\xi, \eta)-2 g_{L}(\xi, \eta)\right| \leq \bar{C}_{0}\left\{|\xi-\eta|^{-2}+|\xi|^{-2 / 3}|\eta|^{-2 / 3}\right\}
$$

Remark 1.8. From (1.2) in Theorem 1.1, we can show that there exists a constant $C$ such that for $\xi, \eta \in C^{*} \backslash L$,

$$
G_{L}(\xi, \eta) \leq C\left\{|\xi||\eta|(1 \vee|\xi-\eta|)^{-2}+\left(\frac{|\xi||\eta|}{|\xi|^{2}+|\eta|^{2}}\right)^{2 / 3}\right\}
$$

For $\xi, \eta \in C^{*} \backslash L$ with $\xi \neq \eta$, we write

$$
g_{L}(\xi, \eta)=\left(\frac{|\xi||\eta|}{|\xi|^{2}+|\eta|^{2}}\right)^{2 / 3} \tilde{M}(\xi, \eta)
$$

Then $\tilde{M}(\xi, \eta)$ is bounded from above by a constant multiple of

$$
\left(\frac{|\xi||\eta|}{|\xi-\eta|^{2}}\right)^{1 / 3}\left(\frac{|\xi|^{2}+|\eta|^{2}}{|\xi-\eta|^{2}}\right)^{2 / 3}+1
$$

For $\delta \in(0, \pi / 2)$, we restrict $g_{L}$ to $\{(\xi, \eta) \in C \times C:-(\pi / 2)+\delta<\arg (\xi), \arg (\eta)<$ $\pi-\delta\}$, then $\tilde{M}(\xi, \eta)$ is bounded from below by a positive constant (depending on $\delta$ ). With the help of these bounds of $g_{L},(1.23)$ gives the upper bound of $G_{L}$ that is the same bound as (1.24) and also gives a lower bound of $G_{L}$.

\section{Preliminary lemmas}

In this section we give preliminary lemmas to calculate bounds of $H_{L}$; in particular we introduce relations among $H_{L}, H_{B}, h_{L}, h_{B}$, fundamental in our proof of Theorem 1.1, which connect the discrete object $H_{L}$ with the continuum one $h_{L}$. As mentioned in the introduction, the estimate (1.7) plays a crucial role to obtain a result so sharp as given in Theorem 1.1. To exploit (1.7) effectively, it is important to suitably define a continuum extension $\tilde{H}_{B}(x,-z)$ of $H_{B}(k,-l)$ as given in the first subsection below.

\subsection{Fundamental relations among $H_{L}, h_{L}, H_{B}, h_{B}$ and $\gamma$} $l \in N$,

The strong Markov property shows that for $\xi \in C^{*} \backslash L$ with $\mathfrak{I}(\xi)>0$ and

$$
H_{L}(\xi,-l)=H_{B}(\xi,-l)+\sum_{j=1}^{\infty} H_{B}(\xi, j) H_{L}(j,-l)
$$

and that for $\xi \in C^{*} \backslash L$ with $\mathfrak{R}(\xi)>0$ and $l \in N$, 


$$
H_{L}(\xi,-l)=\sum_{j=1}^{\infty} H_{\hat{B}}(\xi, i j) H_{L}(i j,-l)
$$

where $\hat{B}=\left\{\zeta \in C^{*}: \mathfrak{R}(\zeta) \leq 0\right\}$. By these equalities and by symmetry, i.e., $H_{\hat{B}}(k, i j)=H_{B}(i k, j)=H_{B}(i k,-j), H_{L}(k,-l)$ may be written as

$$
H_{L}(k,-l)=H_{B}^{2}(i k, l)+\sum_{j=1}^{\infty} H_{B}^{2}(i k, j) H_{L}(j,-l) .
$$

Here $H_{B}^{2}(i k, l)$ and in general $H_{B}^{n}(i k, l) n=1,2, \ldots$ are defined by $H_{B}^{1}(i k, l)=$ $H_{B}(i k, l)$ and

$$
H_{B}^{n}(i k, l)=\sum_{j=1}^{\infty} H_{B}^{n-1}(i k, j) H_{B}(i j, l)=\sum_{j=1}^{\infty} H_{B}(i k, j) H_{B}^{n-1}(i j, l) .
$$

Iterating (2.3) we obtain

$$
H_{L}(k,-l)=\sum_{n=1}^{\infty} H_{B}^{2 n}(i k, l)
$$

Put

$$
\langle x\rangle=k \quad \text { if } k-1 / 2 \leq x<k+1 / 2,
$$

and define $\tilde{H}_{B}^{n}(i x, z)$, an extension of $H_{B}^{n}$ to a function on $\{(i x, z): x>0, z>0\}$, by

$$
\tilde{H}_{B}^{n}(i x, z)=\left\{\begin{aligned}
H_{B}^{n}(i\langle x\rangle,\langle z\rangle), & \text { if } x \geq 1 / 2, z \geq 1 / 2 \\
0, & \text { if } 0<x<1 / 2 \text { or } 0<z<1 / 2 .
\end{aligned}\right.
$$

Then

$$
\tilde{H}_{B}^{n}(i x, z)=\int_{0}^{\infty} \tilde{H}_{B}^{n-1}(i x, y) \tilde{H}_{B}(i y, z) d y .
$$

Let $\tilde{H}_{L}$ be a similar extension of $H_{L}$, or what is the same thing

$$
\tilde{H}_{L}(x,-z)=\sum_{n=1}^{\infty} \tilde{H}_{B}^{2 n}(i x, z) \text {. }
$$

Similarly

$$
h_{L}(x,-z)=\sum_{n=1}^{\infty} h_{B}^{2 n}(i x, z)
$$

Here $h_{B}^{n}(i x, z)$ is defined by $h_{B}^{1}(i x, z)=h_{B}(i x, z)$ and

$$
h_{B}^{n}(i x, z)=\int_{0}^{\infty} h_{B}^{n-1}(i x, y) h_{B}(i y, z) d y .
$$


Remark 2.1. It is the following estimate for $\xi \in C^{*} \backslash L$ and $l \in N$

$$
\left|H_{L}(\xi,-l)-H_{L}(\xi,-l-1)\right| \leq C\left(|\xi+l|^{-1} \vee l^{-1}\right) H_{L}(\xi,-l)
$$

( $C$ is a suitable constant) that is mentioned in Remark 1.5. It is immediate from (1.7) that $\left|H_{B}(\xi,-l)-H_{B}(\xi,-l-1)\right|$ is bounded by a constant multiple of $|\xi+l|^{-1} H_{B}(\xi,-l)$. By $(2.4)$ we have

$$
H_{L}(k,-l)=H_{B}^{2}(i k, l)+\sum_{j=1}^{\infty} H_{L}(k,-j) H_{B}^{2}(i j, l)
$$

which shows that for a constant $C$

$$
\left|H_{L}(k,-l)-H_{L}(k,-l-1)\right| \leq \frac{C}{l} H_{L}(k,-l) .
$$

Combining this inequality with (2.1) and (2.2) yields (2.7). define

For measurable functions $F_{1}$ and $F_{2}$ from $(0,+\infty) \times(-\infty, 0)$ to $[0,+\infty)$, we

$$
F_{1} F_{2}(x,-z)=\int_{0}^{\infty} F_{1}(x,-y) F_{2}(y,-z) d y .
$$

When we use this notation, we regard $h_{L}$ as the function restricted on $(0,+\infty) \times$ $(-\infty, 0)$.

Lemma 2.1. For $x>0$ and $z>0$,

$$
\begin{aligned}
& \left(\tilde{H}_{L}-h_{L}\right)(x,-z)=\left(\gamma+\gamma \tilde{H}_{L}+h_{L} \gamma+h_{L} \gamma \tilde{H}_{L}\right)(x,-z), \\
& \left(\tilde{H}_{L}-h_{L}\right)(x,-z)=\left(\gamma+\gamma h_{L}+\tilde{H}_{L} \gamma+\tilde{H}_{L} \gamma h_{L}\right)(x,-z),
\end{aligned}
$$

where

$$
\gamma(x,-z)=\tilde{H}_{B}^{2}(i x, z)-h_{B}^{2}(i x, z)
$$

Proof. Let

$$
\gamma_{1}(x,-z):=\tilde{H}_{B}^{2}(i x, z) \text { and } \gamma_{2}(x,-z):=h_{B}^{2}(i x, z) .
$$

$\gamma_{1}$ and $\gamma_{2}$ are functions from $(0,+\infty) \times(-\infty, 0)$ to $[0,+\infty)$ and satisfy $\gamma=$ $\gamma_{1}-\gamma_{2}$. We rewrite (2.5) and (2.6) in the form

$$
\begin{gathered}
\tilde{H}_{L}(x,-z)=\gamma_{1}(x,-z)+\gamma_{1} \tilde{H}_{L}(x,-z), \\
h_{L}(x,-z)=\gamma_{2}(x,-z)+h_{L} \gamma_{2}(x,-z) .
\end{gathered}
$$

It is immediate to deduce the following three identities

$$
\begin{gathered}
\tilde{H}_{L}-h_{L}=\gamma+\gamma_{1} \tilde{H}_{L}-h_{L} \gamma_{2}, \\
\gamma_{1} \tilde{H}_{L}=\gamma \tilde{H}_{L}+h_{L} \tilde{H}_{L}-h_{L} \gamma_{2} \tilde{H}_{L},
\end{gathered}
$$


and

$$
h_{L} \gamma_{2}=-h_{L} \gamma+h_{L} \tilde{H}_{L}-h_{L} \gamma_{1} \tilde{H}_{L}
$$

Substituting the latter two into the right-hand side of the first, we get (2.8). We rewrite (2.5) and (2.6) in the form $\tilde{H}_{L}=\gamma_{1}+\tilde{H}_{L} \gamma_{1}$ and $h_{L}=\gamma_{2}+\gamma_{2} h_{L}$. If we write down the decompositions of $\tilde{H}_{L} \gamma_{1}$ and $\gamma_{2} h_{L}$ instead of $\gamma_{1} \tilde{H}_{L}$ and $h_{L} \gamma_{2}$ in the above, we obtain (2.9). The proof of Lemma 2.1 is complete.

\subsection{Estimates of $h_{L}$}

For $\xi \in C \backslash L$ and $z>0, h_{L}(\xi,-z)$ is expressed as

$$
\begin{aligned}
h_{L}(\xi,-z)= & \frac{4}{9 \pi} \sum_{m=1}^{\infty}(-1)^{m+1} m\left(\frac{|\xi| z}{|\xi|^{2}+z^{2}}\right)^{v_{m}} \frac{1}{z} \\
& \cdot\left\{\frac{1}{v_{m}}+\left(\frac{|\xi| z}{|\xi|^{2}+z^{2}}\right)^{2}+\sum_{n=2}^{\infty} c\left(v_{m}, n\right)\left(\frac{|\xi| z}{|\xi|^{2}+z^{2}}\right)^{2 n}\right\} \\
& \cdot \sin \frac{m(2 \theta+\pi)}{3} .
\end{aligned}
$$

Here $v_{m}=2 m / 3, c(v, n)=(v+2 n-1)(v+2 n-2) \cdots(v+n+1) / n !$ and $(|\xi|, \theta)$ is the polar coordinate of $\xi,-\pi / 2<\theta<\pi$. This expression is shown by the usual eigenfunction expansion of a harmonic function. We give a proof of (2.10) in the appendix. We have the following consequences of this expression.

Lemma 2.2. There exist constants $c_{2}$ and $c_{3}$ such that for $z>0$ and $\xi \in C \backslash L$,

$$
h_{L}(\xi,-z) \leq c_{2}\left\{1+\frac{|\xi|^{2}+z^{2}}{|\xi+z|^{2}}\right\} h(|\xi|,-z),
$$

and that for $z>0$ and $\xi \in C \backslash L$ with $|\xi| \in(0, z / 2) \cup(3 z / 2, \infty)$,

$$
\left|h_{L}(\xi,-z)-c_{0} h(|\xi|,-z) \frac{2}{\sqrt{3}} \sin \left(\frac{2 \theta+\pi}{3}\right)\right| \leq c_{3} h(|\xi|,-z)\left(\frac{z}{|\xi|} \wedge \frac{|\xi|}{z}\right)^{2 / 3}
$$

where

$$
h(x,-z)=\left(\frac{x}{x^{2}+z^{2}}\right)^{2 / 3}\left(\frac{1}{z}\right)^{1 / 3}
$$

$(|\xi|, \theta)$ is the polar coordinate of $\xi$ and $c_{0}=(2 / 3 \pi) \sin (\pi / 3)$ (the same constant as in Theorem 1.5). Moreover, for each $\delta \in(0, \pi / 2)$, there exists a positive constant $c(\delta)$ such that if $z>0, \xi \in C \backslash L$ and $-(\pi / 2)+\delta<\arg (\xi)<\pi-\delta$, then

$$
\frac{1}{c(\delta)} h(|\xi|,-z) \leq h_{L}(\xi,-z) \leq c(\delta) h(|\xi|,-z) \text {. }
$$


Proof. To prove (2.12), we calculate $c(v, n)$. By using Stirling's formula, we obtain that for $v \geq 0$ and $n \geq 2$,

$$
\begin{aligned}
c(v, n) & \leq c_{4} \frac{(v+2 n)^{v+2 n}}{(v+n)^{v+n} n^{n}} \frac{1}{\sqrt{v+2 n}} \frac{1}{\sqrt{v+n}} \frac{1}{\sqrt{n}} \\
& =c_{4}\left(t^{t} \cdot(1-t)^{1-t}\right)^{-v-2 n} \frac{1}{\sqrt{v+2 n}} \frac{1}{\sqrt{v+n}} \frac{1}{\sqrt{n}}, \quad t:=\frac{v+n}{v+2 n},
\end{aligned}
$$

where $c_{4}$ is a constant (independent of $v$ and $n$ ). The function $f(t):=t \log t+$ $(1-t) \log (1-t), 0<t<1$, attains the minimum $\log (1 / 2)$ at $t=1 / 2$. Hence

This inequality implies that

$$
c(v, n) \leq 2^{v+2 n} c_{4} \times \frac{1}{(v+n) \sqrt{n}} .
$$

$$
\left|h_{L}(\xi,-z)-c_{0} h(|\xi|,-z) \frac{2}{\sqrt{3}} \sin \left(\frac{2 \theta+\pi}{3}\right)\right| \leq C\left(\frac{|\xi| z}{|\xi|^{2}+z^{2}}\right)^{4 / 3} \frac{1}{z} I(|\xi|, z)
$$

where

$$
\begin{aligned}
I(x, z)= & \frac{4}{9 \pi}\left[\left(\frac{x z}{x^{2}+z^{2}}\right)^{2-2 / 3}+\sum_{n=2}^{\infty} 2^{4 / 3}\left(\frac{2 x z}{x^{2}+z^{2}}\right)^{2 n-2 / 3}\right. \\
& \left.+\sum_{m=2}^{\infty} m\left(\frac{x z}{x^{2}+z^{2}}\right)^{v_{m}-4 / 3}\left\{\frac{1}{v_{m}}+\left(\frac{x z}{x^{2}+z^{2}}\right)^{2}+\sum_{n=2}^{\infty} 2^{v_{m}}\left(\frac{2 x z}{x^{2}+z^{2}}\right)^{2 n}\right\}\right] .
\end{aligned}
$$

If $|\xi| \in(0, z / 2) \cup(3 z / 2, \infty)$, then $2|\xi| z /\left(|\xi|^{2}+z^{2}\right) \leq 12 / 13$, so that $I(|\xi|, z) \leq C$. This gives the asserted bound of $h_{L}(\xi,-z)$.

From (2.12), it is easy to see that there exists a constant $c_{5}$ such that for $z>0$ and $\xi \in C \backslash L$ with $|\xi| \in(0, z / 2) \cup(3 z / 2, \infty)$,

$$
h_{L}(\xi,-z) \leq c_{5} h(|\xi|,-z) \text {. }
$$

To complete the proof of (2.11), it is sufficient to show (2.11) for $z / 2 \leq|\xi| \leq$ $3 z / 2$. Assume $\xi \in C \backslash L$ with $\mathfrak{R}(\xi) \leq \mathfrak{I}(\xi)$. By the strong Markov property, we obtain that

$$
h_{L}(\xi,-z)=h_{B}(\xi,-z)+\int_{0}^{\infty} h_{B}(\xi, y) h_{L}(y,-z) d y .
$$

It is clear that $h_{B}(\xi,-z)$ is bounded by $|\xi| /\left(\pi|\xi+z|^{2}\right)$. We decompose the second term on the right-hand side above into two parts, one the integral of $h_{B}(\xi, y) h_{L}(y,-z)$ over $(0, z / 2) \cup(3 z / 2,+\infty)$ with respect to $y$ and the other that over $[z / 2,3 z / 2]$. (2.14) implies that the former is at most the integral of $c_{5} h_{B}(\xi, y) h(y,-z)$ over $(0,+\infty)$. This is bounded by a constant multiple of $z^{-1}$ as shown by the inequality

$$
\pi h_{B}(\xi, y) h(y,-z) \leq \sqrt{2}|\xi|^{-1} y^{2 / 3} z^{-5 / 3} I_{(0,2|\xi|]}(y)+2 y^{-5 / 3} z^{-1 / 3} I_{(2|\xi|,+\infty)}(y)
$$


that follows from

$$
|\xi-y| \geq \frac{|\xi|}{\sqrt{2}} I_{(0,2|\xi|]}(y)+\frac{y}{2} I_{(2|\xi|,+\infty)}(y)
$$

for $\mathfrak{R}(\xi) \leq \mathfrak{I}(\xi)$. From the expression $(2.10), z h_{L}(y,-z)$ is symmetric, i.e., $z h_{L}(y,-z)=y h_{L}(z,-y)$. This implies that for $z / 2 \leq y \leq 3 z / 2, h_{L}(y,-z) \leq$ $(3 / 2) h_{L}(z,-y)$. Using this bound and noticing that $h_{L}(z,-y)$ is a probability density, the latter is bounded by a constant multiple of $z^{-1}$. Since $|\xi| \in[z / 2,3 z / 2]$, we can replace $z^{-1}$ by a constant multiple of $h(|\xi|,-z)$. Assume $\xi \in C \backslash L$ with $\mathfrak{R}(\xi)>\mathfrak{I}(\xi)$. By the strong Markov property,

$$
h_{L}(\xi,-z)=\int_{0}^{\infty} h_{B}(i \bar{\xi}, y) h_{L}(i y,-z) d y .
$$

The bound $h_{L}(i y,-z) \leq C h(y,-z)$, which has already been shown above, implies that the right-hand side above is bounded by a constant multiple of $z^{-1}$ (recall $|\xi| \in[z / 2,3 z / 2])$.

Proof of (2.13). The upper bound is immediate from (2.11). Let $V(\delta)=$ $\{\xi \in C \backslash L:-(\pi / 2)+\delta<\arg (\xi)<\pi-\delta\}$. From (2.12), it is enough to show that for given $n_{0} \in N$ there exists a constant $C\left(\delta, n_{0}\right)$ such that if $\xi \in V(\delta), z>0$ and $(z /|\xi|) \wedge(|\xi| / z) \geq 1 / n_{0}$, then

$$
h_{L}(\xi,-z) \geq C\left(\delta, n_{0}\right) h(|\xi|,-z) .
$$

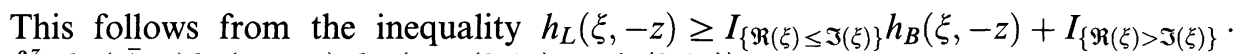
$\int_{z / 2}^{z} h_{B}(i \bar{\xi}, y) h_{B}(i y,-z) d y$ (see (2.15) and (2.17)).

\subsection{Estimates of some elementary integrals}

Let $f$ be a nonnegative measurable function on $(0,+\infty)$ and $u$ and $v$ positive numbers. Then we obtain that

$$
\int_{0}^{\infty}\left(y^{2}+u^{2}\right)^{-2 / 3} f(y) d y \leq v u^{-4 / 3} \int_{0}^{u / v} f(v w) d w+v^{-1 / 3} \int_{u / v}^{\infty} w^{-4 / 3} f(v w) d w .
$$

Let

$$
f_{1}(y)=f_{1}(a, p, v, y):=y^{p} I_{(0, a]}(y)(1 \vee \log (v / y)) .
$$

Assume $-1<p<1 / 3$. We are going to calculate

$$
v u^{-4 / 3} \int_{0}^{u / v} f_{1}(v w) d w+v^{-1 / 3} \int_{u / v}^{\infty} w^{-4 / 3} f_{1}(v w) d w .
$$

Let $a \leq u$ and $e \leq v / a$. Then the second term of (2.18) vanishes and, using that $p>-1$ and performing the integration by parts, the first term is seen to equal

$$
\frac{1}{p+1} u^{-4 / 3}\left\{a^{p+1} \log (v / a)+v^{p+1} \int_{0}^{a / v} w^{p} d w\right\}
$$


hence

$$
\int_{0}^{\infty}\left(y^{2}+u^{2}\right)^{-2 / 3} f_{1}(y) d y \leq K_{p}^{\prime} u^{-4 / 3} a^{p+1} \log (v / a),
$$

where $K_{p}^{\prime}$ is a constant that depends only on $p$. In the case $a \leq u$ and $e>v / a$, the second term of (2.18) also vanishes. We decompose the first term into two parts, one the integral of $u^{-4 / 3} v^{p+1} w^{p} \log (1 / w)$ over $(0,1 / e)$ and the other the integral of $u^{-4 / 3} v^{p+1} w^{p}$ over $[1 / e, a / v]$. Recalling that $p>-1$ and combining this decomposition with (2.19), we obtain that if $a \leq u$,

$$
\int_{0}^{\infty}\left(y^{2}+u^{2}\right)^{-2 / 3} f_{1}(y) d y \leq K_{p}^{\prime \prime} u^{-4 / 3} a^{p+1}(1 \vee \log (v / a))
$$

where $K_{p}^{\prime \prime}$ is another constant depending only on $p$. The case $a>u$ is similarly dealt with; we consider the cases $v / u \leq e, v / a \leq e<v / u$ and $e<v / a$ separately, and as above we use the integration by parts and the assumption that $p<1 / 3$. The second term of $(2.18)$ is at most $(6 /(1-3 p)) u^{p-1 / 3}(1 \vee \log (v / u))$. In consequence we obtain that for $p \in(-1,1 / 3)$,

$$
\int_{0}^{a}\left(y^{2}+u^{2}\right)^{-2 / 3} y^{p}(1 \vee \log (v / y)) d y \leq K_{p} u^{-4 / 3}\left(a^{p+1} \wedge u^{p+1}\right)(1 \vee \log (v /(a \wedge u)))
$$

where $v$ is an arbitrary positive number and $K_{p}$ is a constant depending only on $p$. The same argument also shows that for $p<-1$,

$$
\begin{aligned}
\int_{a}^{\infty}\left(y^{2}+u^{2}\right)^{-2 / 3} y^{p}(1 \vee \log (y / v)) d y & \\
& \leq \begin{cases}K_{p} a^{p-1 / 3}(1 \vee \log (a / v)) & (a \geq u) \\
K_{p} u^{-4 / 3}\left\{a^{p+1}(1 \vee \log (a / v))+u^{p+1}(1 \vee \log (u / v))\right\} & (a<u)\end{cases}
\end{aligned}
$$

and that for $p \in(-1,1 / 3)$,

$$
\int_{0}^{a}\left(y^{2}+u^{2}\right)^{-2 / 3} y^{p} d y \leq K_{p} u^{-4 / 3}\left(a^{p+1} \wedge u^{p+1}\right)
$$

and for $p<-1$,

$$
\int_{a}^{\infty}\left(y^{2}+u^{2}\right)^{-2 / 3} y^{p} d y \leq K_{p} a^{p+1}\left(a^{-4 / 3} \wedge u^{-4 / 3}\right) .
$$

The bounds (2.20), (2.21), (2.22) and (2.23) will be used on several occasions in the succeeding sections.

LEMmA 2.3. There exists a constant $c_{6}$ such that for $x>0$ and $z \geq 1 / 2$,

$$
\left|\tilde{H}_{B}(i x, z)-h_{B}(i x, z)\right| \leq c_{6}\left(x^{2}+z^{2}\right)^{-1},
$$


and that for $k \in N$ and $l \in Z$,

$$
\left|H_{B}(i k, l)-h_{B}(i k, l)\right| \leq c_{6}\left(k^{2}+l^{2}\right)^{-3 / 2} .
$$

Proof. The second assertion is a restatement of (1.7). The order $O\left(\left(x^{2}+z^{2}\right)^{-1}\right)$ of the first comes from the difference between $h_{B}(i\langle x\rangle,\langle z\rangle)$ and $h_{B}(i x, z)$.

\subsection{Estimates of $\gamma$}

In this subsection we derive estimates for $\gamma(x,-z)$. In the first two preliminary lemmas we give some estimate of $\gamma(x,-z)$ by using the first inequality of Lemma 2.3. If $x$ and $z$ are elements of $\mathbb{N}$, then we deduce a sharp estimate of $\gamma(x,-z)$ (Lemma 2.6) from (1.7). In its proof it will be revealed how important the manner of the definition of $\tilde{H}_{B}$ is for exploiting the relation (1.7) effectively.

LEMMA 2.4. There is a constant $c_{7}$ such that for $z \geq 1 / 2$ and $x \geq z$,

$$
|\gamma(x,-z)| \leq \frac{c_{7}}{x z}
$$

and that for $z \geq 1 / 2$ and $x \in(0, z)$,

$$
|\gamma(x,-z)| \leq \frac{c_{7}}{z^{2}}(1 \vee \log (z /(x \vee 1))) .
$$

Moreover, there exists a constant $c_{8}$ such that for $x \geq 1 / 2$ and $z \geq 1 / 2$,

$$
\left|h_{B}^{2}(i x, z)-h_{B}^{2}(i\langle x\rangle, z)\right| \leq \frac{c_{8}}{z^{2}}(1 \vee \log z)
$$

Proof. We write

where

$$
\gamma(x,-z):=\gamma_{3}(x,-z)+\gamma_{4}(x,-z)
$$

$$
\gamma_{3}(x,-z)=\int_{0}^{\infty} \tilde{H}_{B}(i x, y)\left\{\tilde{H}_{B}(i y, z)-h_{B}(i y, z)\right\} d y
$$

and

$$
\gamma_{4}(x,-z)=\int_{0}^{\infty}\left\{\tilde{H}_{B}(l x, y)-h_{B}(i x, y)\right\} h_{B}(\iota y, z) d y .
$$

We apply Lemma 2.3 to see that

$$
\left|\gamma_{3}(x,-z)\right| \leq \int_{1 / 2}^{\infty} h_{B}(i x, y) \frac{c_{6}}{y^{2}+z^{2}} d y+c_{6}^{2}\left(x^{-2} \wedge z^{-2}\right) \int_{1 / 2}^{\infty} \frac{1}{y^{2}} d y .
$$

The second term on the right-hand side above is equal to $2 c_{6}^{2}\left(x^{-2} \wedge z^{-2}\right)$. By replacing $1 /\left(y^{2}+z^{2}\right)$ by $1 / z^{2}$, the first term is at most $\left(c_{6} / 2\right) z^{-2}$. The obvious inequality $h_{B}(i x, y) \leq 1 /(x \pi)$ implies that it is also at most $\left(c_{6} / 2\right) x^{-1} z^{-1}$. Thus $\left|\gamma_{3}(x,-z)\right| \leq C z^{-1}\left(x^{-1} \wedge z^{-1}\right)$. By Lemma 2.3 again, we have 


$$
\left|\gamma_{4}(x,-z)\right| \leq \frac{1}{2 \pi} \int_{0}^{1 / 2} h_{B}(i x, y) \frac{1}{y^{2}+z^{2}} d y+\int_{1 / 2}^{\infty} \frac{c_{6}}{x^{2}+y^{2}} h_{B}(i y, z) d y .
$$

By the same reason as above, the first term on the right-hand side of (2.27) is bounded by a constant multiple of $z^{-1}\left(x^{-1} \wedge z^{-1}\right)$. It is easy to see that

$$
\int_{1 / 2}^{\infty} \frac{1}{x^{2}+y^{2}} \frac{y}{y^{2}+z^{2}} d y \leq \frac{1}{x^{2} z^{2}} \int_{0}^{x} y d y I_{\{x>1 / 2\}}+\int_{x \vee(1 / 2)}^{\infty} \frac{1}{y\left(y^{2}+z^{2}\right)} d y .
$$

The first term on the right-hand side is at most $(1 / 2) z^{-2}$. The second term is equal to

$$
\frac{1}{2 z^{2}} \log \left(1+\left(\frac{z}{x \vee(1 / 2)}\right)^{2}\right)
$$

Hence the second term on the right-hand side of (2.27) is bounded by $3\left(c_{6} / z^{2} \pi\right)$. $(1 \vee \log (z /(x \vee 1))$. By symmetry, we see that the second term on the righthand side of $(2.27)$ is also bounded by $\left(3 c_{6} / \pi\right)(x \vee z)^{-2}(1 \vee \log ((z / x) \vee(x / z)))$.

We notice that for $x \geq 1 / 2$, the difference between $h_{B}(i x, y)$ and $h_{B}(i\langle x\rangle, y)$ is bounded by a constant multiple of $\left(x^{2}+y^{2}\right)^{-1}$. The definition of $h_{B}^{2}$ implies that for $x \geq 1 / 2$ and $z \geq 1 / 2$,

$$
\left|h_{B}^{2}(i x, z)-h_{B}^{2}(i\langle x\rangle, z)\right| \leq C \int_{0}^{\infty} \frac{1}{x^{2}+y^{2}} h_{B}(i y, z) d y .
$$

We have the desired estimate for $\left|h_{B}^{2}(i x, z)-h_{B}^{2}(i\langle x\rangle, z)\right|$ by the same argument as showing that the second term on the right-hand side of (2.27) is bounded by $\left(3 c_{6} / \pi\right)(x \vee z)^{-2}(1 \vee \log ((z / x) \vee(x / z)))$. The proof of Lemma 2.4 is complete.

LEMMA 2.5. There exists a constant $c_{9}$ such that

$$
|\gamma(x,-z)| \leq c_{9} z^{-1} \text { for } z \in(0,1 / 2) \text { and } x \in(0,1)
$$

and

$$
|\gamma(x,-z)| \leq c_{9} x^{-1} \log \left(\frac{x}{z}\right) \quad \text { for } z \in(0,1 / 2) \text { and } \quad x \geq 1
$$

Proof. If $z \in(0,1 / 2)$ then

$$
-\gamma(x,-z)=\int_{0}^{\infty} h_{B}(i x, y) h_{B}(i y, z) d y .
$$

By change of variables $u=(y / x)^{2}$, this integral is seen to equal

$$
h_{B}^{2}(i x, z)=\frac{1}{2 \pi^{2} x} \int_{0}^{\infty} \frac{1}{u+(z / x)^{2}} \frac{1}{u+1} d u=\frac{1}{1-(z / x)^{2}} \frac{1}{\pi^{2} x} \log \left(\frac{x}{z}\right) .
$$

This gives (2.29). (2.28) is immediate from $h_{B}(i y, z) \leq 1 /(z \pi)$. 
Lemma 2.6. There exists a constant $c_{10}$ such that for $k, l \in N$,

$$
|\gamma(k,-l)| \leq c_{10} k^{-1} l^{-2} .
$$

Moreover, there exists a constant $c_{11}$ such that for $x>0$ and $k \in N$,

$$
\begin{gathered}
\left|\int_{k-1 / 2}^{k+1 / 2} \gamma(x,-y) d y-\gamma(x,-k)\right| \leq c_{11} x^{-1} k^{-2}, \\
\left|\int_{k-1 / 2}^{k+1 / 2} \gamma(y,-x) d y-\gamma(k,-x)\right| \leq c_{11} k^{-1} x^{-1}\left(k^{-1} \wedge x^{-1}\right) .
\end{gathered}
$$

Proof. The proof of (2.31) is carried out by estimating $\gamma_{3}$ and $\gamma_{4}$ that are defined in the proof of Lemma 2.4. We first prove that

$$
\left|\gamma_{4}(k,-l)\right| \leq C k^{-1} l^{-2}
$$

where $C$ is a suitable constant. From the definition of $\gamma_{4}$, we obtain

$$
\left|\gamma_{4}(k,-l)\right| \leq \int_{0}^{1 / 2} h_{B}(i k, y) h_{B}(i y, l) d y+\left|\int_{1 / 2}^{\infty}\left(\tilde{H}_{B}(i k, y)-h_{B}(i k, y)\right) h_{B}(i y, l) d y\right| .
$$

The first term on the right-hand side is at most $\left(1 / 8 \pi^{2}\right) k^{-1} l^{-2}$. To estimate the second term, we make the decomposition

$$
\left\{\tilde{H}_{B}(i k, y)-h_{B}(i k, y)\right\} h_{B}(i y, l)=I_{1}(k, y, l)+I_{2}(k, y, l)+I_{3}(k, y, l) .
$$

Here

$$
\begin{aligned}
& I_{1}(k, y, l)=\left\{H_{B}(i k,\langle y\rangle)-h_{B}(i k, y)\right\}\left\{h_{B}(i y, l)-h_{B}(i\langle y\rangle, l)\right\}, \\
& I_{2}(k, y, l)=\left\{H_{B}(i k,\langle y\rangle)-h_{B}(i k,\langle y\rangle)\right\} h_{B}(i\langle y\rangle, l), \\
& I_{3}(k, y, l)=\left\{h_{B}(i k,\langle y\rangle)-h_{B}(i k, y)\right\} h_{B}(i\langle y\rangle, l) .
\end{aligned}
$$

According to Lemma 2.3, $\left|I_{1}(k, y, l)\right|$ and $\left|I_{2}(k, y, l)\right|$ are bounded by a constant multiple of $\left(1 /\left(k^{2}+y^{2}\right)\right) l^{-2}$. Moreover, the integral of $1 /\left(k^{2}+y^{2}\right)$ over $(0, \infty)$ with respect to $y$ is equal to $(\pi / 2) k^{-1}$. For the proof of $(2.34)$, it suffices to show that

$$
\left|\int_{1 / 2}^{\infty} I_{3}(k, y, l) d y\right| \leq C k^{-1} l^{-2}
$$

where $C$ is a suitable constant. From Taylor's series, we obtain that for $x>0$, $j \in \boldsymbol{Z}$ and $|y-j| \leq 1 / 2$,

$$
h_{B}(i x, y)-h_{B}(i x, j)=\frac{\partial}{\partial y} h_{B}(i x, j)(y-j)+\frac{1}{2} \frac{\partial^{2}}{\partial y^{2}} h_{B}(i x, j+\theta)(y-j)^{2},
$$

with $|\theta| \leq 1 / 2$. The integral of the first term on the right-hand side over $[j-1 / 2, j+1 / 2)$ with respect to $y$ vanishes. Moreover, $\left|\left(\partial^{2} / \partial y^{2}\right) h_{B}(i x, y)\right| \leq$ 
$(8 / \pi)\left(x^{2}+y^{2}\right)^{-3 / 2}$. Thus for $x>0$ and $j \in Z$,

$$
\left|\int_{J-1 / 2}^{J+1 / 2} h_{B}(i x, y) d y-h_{B}(i x, j)\right| \leq \frac{8}{3 \pi\left(x^{2}+j^{2}\right)^{3 / 2}} .
$$

This implies (2.35).

Next we prove

$$
\left|\gamma_{3}(k,-l)\right| \leq C k^{-1} l^{-2}
$$

with a suitable constant $C$. The proof is similar to that of (2.34). We make the decomposition

$$
\tilde{H}_{B}(i k, y)\left\{\tilde{H}_{B}(i y, l)-h_{B}(i y, l)\right\}=I_{4}(k, y, l)+I_{5}(k, y, l) .
$$

Here

$$
\begin{aligned}
& I_{4}(k, y, l)=H_{B}(i k,\langle y\rangle)\left\{H_{B}(i\langle y\rangle, l)-h_{B}(i\langle y\rangle, l)\right\}, \\
& I_{5}(k, y, l)=H_{B}(i k,\langle y\rangle)\left\{h_{B}(i\langle y\rangle, l)-h_{B}(l y, l)\right\} .
\end{aligned}
$$

By Lemma 2.3,

$$
\begin{aligned}
\int_{1 / 2}^{\infty}\left|I_{4}(k, y, l)\right| d y & \leq c_{6}\left(c_{6}+\frac{1}{\pi}\right) \sum_{j=1}^{\infty} k^{-1} l^{-1} \frac{1}{j^{2}+l^{2}} \\
& \leq c_{6}\left(c_{6}+\frac{1}{\pi}\right) \frac{\pi}{2} k^{-1} l^{-2} .
\end{aligned}
$$

The same argument as verifying (2.36) gives

$$
\left|\int_{J-1 / 2}^{J+1 / 2} h_{B}(i y, x) d y-h_{B}(i j, x)\right| \leq \frac{8}{3 \pi\left(j^{2}+x^{2}\right)^{3 / 2}} .
$$

Hence as above we obtain that

$$
\left|\int_{1 / 2}^{\infty} I_{5}(k, y, l) d y\right| \leq \frac{4}{3}\left(c_{6}+\frac{1}{\pi}\right) k^{-1} l^{-2}
$$

proving (2.31).

Notice that

$$
\int_{k-1 / 2}^{k+1 / 2} \gamma(x,-y) d y-\gamma(x,-k)=\int_{0}^{\infty} h_{B}(i x, \tilde{y})\left\{h_{B}(i \tilde{y}, k)-\int_{k-1 / 2}^{k+1 / 2} h_{B}(i \tilde{y}, y) d y\right\} d \tilde{y}
$$

and apply (2.36). Then we have (2.32). Similarly

$$
\left|\int_{k-1 / 2}^{k+1 / 2} \gamma(y,-x) d y-\gamma(k,-x)\right| \leq \frac{8}{3 \pi^{2}} \int_{0}^{\infty} \frac{1}{k^{2}+\tilde{y}^{2}} \cdot \frac{1}{\tilde{y}^{2}+x^{2}} d \tilde{y} .
$$

This implies (2.33). The proof of Lemma 2.6 is complete. 


\section{Asymptotic behavior of $H_{L}(\xi,-l)$}

In this section, we will prove Theorems 1.1, 1.2 and 1.3 and Corollary 1.4.

LEMMA 3.1. There exists a constant $c_{12}$ such that for $x \geq 1 / 2$ and $z \geq 1 / 2$,

$$
\left|\tilde{H}_{L}(x,-z)-h_{L}(x,-z)\right| \leq c_{12} z^{-5 / 3} .
$$

Proof. The proof is carried out by estimating the right-hand side of (2.9). Lemma 2.4 implies that $|\gamma(x,-z)|$ is bounded by a constant multiple of $z^{-2}(1 \vee \log z)$. Since $\tilde{H}_{L}$ is an extension of hitting distribution $H_{L},\left|\tilde{H}_{L} \gamma(x,-z)\right|$ is also bounded by a constant multiple of $z^{-2}(1 \vee \log z)$. We will show that for $x \geq 1 / 2$ and $z \geq 1 / 2$,

$$
\left|\gamma h_{L}(x,-z)\right| \leq \begin{cases}c_{13} x^{-1} z^{-1}(1 \vee \log x) & (x \geq z) \\ c_{13} x^{-1 / 3} z^{-5 / 3} & (x<z)\end{cases}
$$

This gives

$$
\left|\gamma h_{L}(x,-z)\right| \leq\left(6 c_{13} / e\right) z^{-5 / 3} .
$$

Since $\tilde{H}_{L}$ is an extension of hitting distribution $H_{L},\left|\tilde{H}_{L} \gamma h_{L}(x,-z)\right|$ is also bounded by the right-hand side above.

To prove (3.1), we apply (2.11) to obtain

$$
\begin{aligned}
\left|\gamma h_{L}(x,-z)\right| \leq & 2 c_{2} z^{-5 / 3} \int_{0}^{1 / 2}|\gamma(x,-y)| y^{2 / 3} d y \\
& +2 c_{2} \int_{1 / 2}^{\infty}|\gamma(x,-y)| h(y,-z) d y .
\end{aligned}
$$

By virtue of Lemma 2.5, we may replace $|\gamma(x,-y)|$ by $c_{9}\left\{I_{\{x<1\}} y^{-1}+I_{\{x \geq 1\}} x^{-1}\right.$. $\log (x / y)\}$, showing that the first term on the right-hand side of (3.2) is at most $4 c_{2} c_{9} x^{-1} z^{-5 / 3}(1 \vee \log x)$. Hence it is enough to show that

$$
\int_{1 / 2}^{\infty}|\gamma(x,-y)| h(y,-z) d y \leq C x^{-1 / 3} z^{-1}\left(x^{-2 / 3} \wedge z^{-2 / 3}\right) .
$$

We decompose the left-hand side of the above into two parts, one the integral over $[1 / 2, x]$ and the other that over $(x,+\infty)$. By Lemma 2.4, the former is bounded by

$$
c_{7} x^{-1} z^{-1 / 3} \int_{1 / 2}^{x} y^{-1 / 3}\left(y^{2}+z^{2}\right)^{-2 / 3} d y .
$$

The latter is bounded by

$$
c_{7} z^{-1 / 3} \int_{x}^{\infty} y^{-4 / 3}\left(y^{2}+z^{2}\right)^{-2 / 3}(1 \vee \log (y / x)) d y .
$$


Take $p=-1 / 3, a=x, u=z$. By (2.22), the integral in (3.3) is at most $K_{-1 / 3} z^{-4 / 3}\left(x^{2 / 3} \wedge z^{2 / 3}\right)$. Take $p=-4 / 3, a=v=x, u=z$. By $(2.21)$, the integral in (3.4) is at most $3 K_{-4 / 3} x^{-1 / 3}\left(x^{-4 / 3} \wedge z^{-4 / 3}\right)$. These complete the proof of $(3.1)$.

Notice that, in the proof of (3.1), the bound $c_{13} x^{-1} z^{-1}(1 \vee \log x)$ comes from the first term on the right-hand side of (3.2). Then we have the following lemma.

Lemma 3.2. There exists a constant $\hat{c}_{13}$ such that for $x \geq 1 / 2$ and $z \geq 1 / 2$,

$$
\left|\int_{1 / 2}^{\infty} \gamma(x,-y) h_{L}(y,-z) d y\right| \leq \hat{c}_{13} x^{-1 / 3} z^{-1}\left(x^{-2 / 3} \wedge z^{-2 / 3}\right) .
$$

Proposition 3.3. There exists a constant $c_{14}$ such that for $x \geq 1 / 2$ and $z \geq$ $1 / 2$,

$$
\left|\tilde{H}_{L}(x,-z)-h_{L}(x,-z)\right| \leq c_{14} x^{-1 / 3} z^{-4 / 3}\left(x^{-1 / 3} \wedge z^{-1 / 3}\right) .
$$

Proof. We will show that each term $\gamma=\gamma(x,-z), \gamma \tilde{H}_{L}, h_{L} \gamma, h_{L} \gamma \tilde{H}_{L}$, on the right-hand side of (2.8), is bounded in absolute value by a constant multiple of $x^{-1 / 3} z^{-4 / 3}\left(x^{-1 / 3} \wedge z^{-1 / 3}\right)$. Lemma 2.4 implies such an estimate of $|\gamma(x,-z)|$. Notice that $\tilde{H}_{L}(y,-z)=0$ for $0<y<1 / 2$. By Lemmas 2.4 and 3.1 , the difference between $\gamma \tilde{H}_{L}(x,-z)$ and

$$
\int_{1 / 2}^{\infty} \gamma(x,-y) h_{L}(y,-z) d y
$$

is at most

$$
c_{7} c_{12} z^{-5 / 3}\left\{x^{-1} \int_{1 / 2}^{x} y^{-1} d y+\int_{x}^{\infty} y^{-2}(1 \vee \log (y / x)) d y\right\} .
$$

This integral is negligible as $O\left(x^{-1} z^{-5 / 3}(1 \vee \log x)\right)$. The bound for the integral in (3.5) was already given in Lemma 3.2. Hence we have the desired estimate of $\left|\gamma \tilde{H}_{L}(x,-z)\right|$.

Let us show

$$
\left|h_{L} \gamma(x,-z)\right| \leq c_{15} x^{-1 / 6} z^{-4 / 3}\left(x^{-1 / 2} \wedge z^{-1 / 2}\right) \quad(x \geq 1 / 2, z \geq 1 / 2) .
$$

By (2.11), we obtain that

$$
\left|h_{L} \gamma(x,-z)\right| \leq 2 c_{2} x^{-2 / 3} \int_{0}^{1 / 2}|\gamma(y,-z)| y^{-1 / 3} d y+2 c_{2} \int_{1 / 2}^{\infty} h(x,-y)|\gamma(y,-z)| d y .
$$

In view of (2.25), we dominate $|\gamma(y,-z)|$ by $c_{7} z^{-2}(1 \vee \log z)$ to see that the first term on the right-hand side above is at most $6 c_{2} c_{7} x^{-2 / 3} z^{-2}(1 \vee \log z)$. We decompose the second term into two parts, one the integral over $[1 / 2, z)$ and the 
other that over $[z, \infty)$. By Lemma 2.4 , the former is bounded by

$$
2 c_{2} c_{7} x^{2 / 3} z^{-2} \int_{1 / 2}^{z}\left(x^{2}+y^{2}\right)^{-2 / 3} y^{-1 / 3}(1 \vee \log (z / y)) d y .
$$

The latter is bounded by

$$
2 c_{2} c_{7} x^{2 / 3} z^{-1} \int_{z}^{\infty}\left(x^{2}+y^{2}\right)^{-2 / 3} y^{-4 / 3} d y .
$$

Take $p=-1 / 3, a=v=z, u=x$ in (2.20) to see that the integral in (3.7) is at most $K_{-1 / 3} x^{-4 / 3}\left(x^{2 / 3} \wedge z^{2 / 3}\right)(1 \vee \log (z / x))$. Take $p=-4 / 3, a=z, u=x$ in (2.23). The integral in (3.8) is seen to be at most $K_{-4 / 3} z^{-1 / 3}\left(x^{-4 / 3} \wedge z^{-4 / 3}\right)$. Thus we obtain (3.6).

Notice that $\tilde{H}_{L}(y,-z)=0$ for $0<y<1 / 2$. By Lemma 3.1 and (3.6),

$$
\left|h_{L} \gamma \tilde{H}_{L}(x,-z)-\int_{1 / 2}^{\infty} h_{L} \gamma(x,-y) h_{L}(y,-z) d y\right|=O\left(x^{-2 / 3} z^{-5 / 3}\right) .
$$

The same arguments as in the proof of (3.1) and (3.6) prove

$$
\int_{1 / 2}^{\infty}\left|h_{L} \gamma(x,-y)\right| h_{L}(y,-z) d y \leq C x^{-1 / 3} z^{-4 / 3}\left(x^{-1 / 3} \wedge z^{-1 / 3}\right) .
$$

Hence we have the desired estimate of $\left|h_{L} \gamma \tilde{H}_{L}(x,-z)\right|$. The proof of Proposition 3.3 is complete.

If $x, z \in N$, then the estimate given in Proposition 3.3 can be improved significantly.

Proposition 3.4. There exists a constant $c_{16}$ such that for $k, l \in N$,

$$
\left|H_{L}(k,-l)-h_{L}(k,-l)\right| \leq c_{16} k^{-2 / 3} l^{-5 / 3} \text {. }
$$

Proof. We will show that each term $\gamma, \gamma h_{L}, \tilde{H}_{L} \gamma, \tilde{H}_{L} \gamma h_{L}$, appearing in the right-hand side of (2.9), is bounded in absolute value by a constant multiple of $k^{-2 / 3} l^{-5 / 3}$. The estimate of $|\gamma(k,-l)|$ is immediate from $(2.31)$.

We prove that there exists a constant $C$ such that for $k, l \in N$,

$$
\left|\tilde{H}_{L} \gamma(k,-l)\right| \leq C k^{-2 / 3} l^{-2} \text {. }
$$

We decompose

$$
\begin{aligned}
\tilde{H}_{L} \gamma(k,-l)= & \sum_{j=1}^{\infty} H_{L}(k,-j)\left\{\int_{J-1 / 2}^{j+1 / 2} \gamma(y,-l) d y-\gamma(j,-l)\right\} \\
& +\sum_{j=1}^{\infty} H_{L}(k,-j) \gamma(j,-l) .
\end{aligned}
$$


From Lemma $2.6,\left|\tilde{H}_{L} \gamma(k,-l)\right|$ is bounded by a constant multiple of $l^{-2} \sum_{J=1}^{\infty}$. $H_{L}(k,-j) j^{-1}$. The bound (3.9) therefore follows if we show

$$
\sum_{j=1}^{\infty} H_{L}(k,-j) j^{-1} \leq C k^{-2 / 3}
$$

But this is immediately seen by writing $H_{L}=\left(H_{L}-h_{L}\right)+h_{L}$ and applying Proposition 3.3 and (2.11). Thus we obtain (3.9).

To estimate $\gamma h_{L}(k,-l)$, we decompose

$$
\gamma h_{L}(k,-l)=\int_{0}^{1 / 2} \gamma(k,-y) h_{L}(y,-l) d y+\int_{1 / 2}^{\infty} \gamma(k,-y) h_{L}(y,-l) d y .
$$

The first term on the right-hand side above is bounded by $4 c_{2} c_{9} k^{-1} l^{-5 / 3}$. $(1 \vee \log k$ ) (see the bound for the first term on the right-hand side of (3.2)). In the proof of Proposition 3.3, we proved that the difference between the second term and $\gamma \tilde{H}_{L}(k,-l)$ is bounded by a constant multiple of $k^{-1} l^{-5 / 3}(1 \vee \log k)$. Let us show that there exists a constant $C$ such that for $k, l \in N$,

$$
\left|\gamma \tilde{H}_{L}(k,-l)\right| \leq C k^{-1} l^{-5 / 3} .
$$

The proof is similar to that of (3.9). In view of Lemma 2.6, it is enough to show that

$$
\sum_{j=1}^{\infty} j^{-2} H_{L}(j,-l) \leq C l^{-5 / 3}
$$

This is also seen by writing $H_{L}=\left(H_{L}-h_{L}\right)+h_{L}$ and applying Proposition 3.3 and (2.11). We accordingly conclude that

$$
\left|\gamma h_{L}(k,-l)\right| \leq c_{17} k^{-1} l^{-5 / 3}(1 \vee \log k) .
$$

We will show that

$$
\left|\int_{J-1 / 2}^{j+1 / 2} \gamma h_{L}(y,-l) d y-\gamma h_{L}(j,-l)\right| \leq c_{18} j^{-1} l^{-5 / 3}
$$

This bound and (3.11) give

$$
\left|\tilde{H}_{L} \gamma h_{L}(k,-l)\right| \leq\left(c_{17}+c_{18}\right) l^{-5 / 3} \sum_{j=1}^{\infty} H_{L}(k,-j) j^{-1}(1 \vee \log j) .
$$

Moreover, the same arguments as in the proof of (3.9) show that the sum on the right-hand side above is bounded by a constant multiple of $k^{-2 / 3}$.

To prove (3.12), we rewrite the left-hand side of (3.12) in the form

$$
\left|\int_{0}^{\infty}\left\{\int_{J-1 / 2}^{\jmath+1 / 2} \gamma(\tilde{y},-y) d \tilde{y}-\gamma(j,-y)\right\} h_{L}(y,-l) d y\right| .
$$


Apply (2.11) and (2.33) to see that the integral above is at most

$$
2 c_{2} c_{11} j^{-1} l^{-5 / 3} \int_{0}^{\infty} y^{-1 / 3}\left(j^{-1} \wedge y^{-1}\right) d y
$$

Thus we have (3.12). The proof of Proposition 3.4 is complete.

Proposition 3.5. There exists a constant $\hat{C}_{0}$ such that for $x+i y \in C \backslash L$ with $x \vee y \geq 1 / 2$ and $z \geq 1 / 2$,

$$
\begin{aligned}
& \left|H_{L}(\langle x\rangle+i\langle y\rangle,-\langle z\rangle)-h_{L}(x+i y,-z)\right| \\
& \quad \leq \hat{C}_{0}\left\{|x+i y+z|^{-2}+|x+i y|^{-1 / 3} z^{-4 / 3}\left(|x+i y|^{-1 / 3} \wedge z^{-1 / 3}\right)\right\}
\end{aligned}
$$

Proof. We consider the case $x \leq y$ and $y \geq 1 / 2$. To estimate the difference $H_{L}(\langle x\rangle+i\langle y\rangle,-\langle z\rangle)-h_{L}(x+i y,-z)$, we recall (2.1) and use the relation

$$
h_{L}(x+i y,-z)=h_{B}(x+i y,-z)+\int_{0}^{\infty} h_{B}(x+i y, \tilde{y}) h_{L}(\tilde{y},-z) d \tilde{y} .
$$

The term $\hat{C}_{0}|x+i y+z|^{-2}$ on the right-hand side of (3.13) comes from the difference between $H_{B}(\langle x\rangle+i\langle y\rangle,-\langle z\rangle)$ and $h_{B}(x+i y,-z)$.

Let

$$
\begin{aligned}
& h_{1}(x+i y, z)=\int_{1 / 2}^{\infty} H_{B}(\langle x\rangle+i\langle y\rangle,\langle\tilde{y}\rangle)\left\{\tilde{H}_{L}(\tilde{y},-\langle z\rangle)-h_{L}(\tilde{y},-z)\right\} d \tilde{y}, \\
& h_{2}(x+\imath y, z)=\int_{0}^{\infty}\left\{H_{B}(\langle x\rangle+i\langle y\rangle,\langle\tilde{y}\rangle) I_{\{\tilde{y} \geq 1 / 2\}}-h_{B}(x+i y, \tilde{y})\right\} h_{L}(\tilde{y},-z) d \tilde{y} .
\end{aligned}
$$

By the definition of $\langle\tilde{y}\rangle$, the difference between the second term on the righthand side of (2.1) and that of (3.14) is equal to $h_{1}(x+i y, l)+h_{2}(x+i y, l)$. We will show that for $x \leq y$ with $y \geq 1 / 2$ and $z \geq 1 / 2$,

$$
\begin{aligned}
& \left|\sum_{J=1}^{\infty} H_{B}(\langle x\rangle+i\langle y\rangle, j) H_{L}(j,-\langle z\rangle)-\int_{0}^{\infty} h_{B}(x+i y, \tilde{y}) h_{L}(\tilde{y},-z) d \tilde{y}\right| \\
& \quad \leq\left|h_{1}(x+i y, z)\right|+\left|h_{2}(x+i y, z)\right| \\
& \quad \leq C|x+i y|^{-1 / 3} z^{-4 / 3}\left(|x+i y|^{-1 / 3} \wedge z^{-1 / 3}\right)
\end{aligned}
$$

where $C$ is a constant. Using Proposition 3.3, Lemma 2.3 and (2.11) and then recalling $x \leq y$ and $y \geq 1 / 2$, we observe that $\left|h_{1}(x+i y, z)\right|$ and $\left|h_{2}(x+i y, z)\right|$ are bounded by constant multiples of

$$
\int_{1 / 2}^{\infty}|x+i y|\left(|x+i y|^{-2} \wedge \tilde{y}^{-2}\right) \tilde{y}^{-1 / 3} z^{-4 / 3}\left(\tilde{y}^{-1 / 3} \wedge z^{-1 / 3}\right) d \tilde{y}
$$


and of

$$
\begin{aligned}
\int_{0}^{1 / 2} \mid x & +\left.i y\right|^{-1} \tilde{y}^{2 / 3} z^{-5 / 3} d \tilde{y} \\
& +\int_{1 / 2}^{\infty}\left(|x+i y|^{-2} \wedge \tilde{y}^{-2}\right) \tilde{y}^{2 / 3} z^{-1 / 3}\left(\tilde{y}^{-4 / 3} \wedge z^{-4 / 3}\right) d \tilde{y}
\end{aligned}
$$

respectively. The desired bound follows from a simple calculation by dealing with the cases $|x+i y| \leq z$ and $|x+i y|>z$, separately.

It remains to show that (3.13) holds for $x>y$ with $x \geq 1 / 2$. In this case, we use the relations (2.2) and

$$
h_{L}(x+i y,-z)=\int_{0}^{\infty} h_{\hat{B}}(x+i y, i \tilde{y}) h_{L}(i \tilde{y},-z) d \tilde{y} .
$$

Notice that $H_{\hat{B}}(\langle x\rangle+i\langle y\rangle, i j)=H_{B}(\langle y\rangle+i\langle x\rangle, j) \quad$ and $\quad h_{\hat{B}}(x+i y, i \tilde{y})=$ $h_{B}(y+l x, \tilde{y})$. Then

$$
\begin{aligned}
& \left|H_{L}(\langle x\rangle+i\langle y\rangle,-\langle z\rangle)-h_{L}(x+i y,-z)\right| \\
& \quad=\left|\sum_{j=1}^{\infty} H_{B}(\langle y\rangle+\imath\langle x\rangle, j) H_{L}(i j,-\langle z\rangle)-\int_{0}^{\infty} h_{B}(y+i x, \tilde{y}) h_{L}(i \tilde{y},-z) d \tilde{y}\right| .
\end{aligned}
$$

By using the bound

$$
\left.\mid H_{L}(i<\tilde{y}\rangle,-\langle z\rangle\right)-h_{L}(i \tilde{y},-z) \mid \leq \hat{C}^{\prime} \tilde{y}^{-1 / 3} z^{-4 / 3}\left(\tilde{y}^{-1 / 3} \wedge z^{-1 / 3}\right)
$$

which has already been shown above, the same argument as in the proof of (3.15) verifies the estimate (3.13) for $x>y$ with $x \geq 1 / 2$ and $z \geq 1 / 2$.

Proof of Theorem 1.1. Assume $\xi \in C^{*} \backslash L$ and $\mathfrak{R}(\xi) \leq \mathfrak{I}(\xi)$. We use the following decomposition (as in the proof of Proposition 3.5):

$$
H_{L}(\xi,-l)-h_{L}(\xi,-l)=H_{B}(\xi,-l)-h_{B}(\xi,-l)+h_{1}(\xi,-l)+h_{2}(\xi,-l),
$$

where $h_{1}$ and $h_{2}$ are defined in the proof of Proposition 3.5. The term $C_{0}|\xi+l|^{-3}$ on the right-hand side of (1.2) comes from the difference between $H_{B}(\xi,-l)$ and $h_{B}(\xi,-l)$. We will show that for $\xi \in C^{*} \backslash L$ with $\mathfrak{R}(\xi) \leq \mathfrak{I}(\xi)$,

$$
\begin{gathered}
\left|\sum_{j=1}^{\infty} H_{B}(\xi, j) H_{L}(j,-l)-\int_{0}^{\infty} h_{B}(\xi, y) h_{L}(y,-l) d y\right| \\
=\left|h_{1}(\xi, l)+h_{2}(\xi, l)\right| \leq C|\xi|^{-2 / 3} l^{-5 / 3},
\end{gathered}
$$

where $C$ is a constant. The proof of (3.17) is similar to (2.31). Put

$$
I_{6}(\xi, j, l)=H_{B}(\xi, j)\left\{H_{L}(j,-l)-h_{L}(j,-l)\right\} \text {, }
$$

and 


$$
I_{7}(\xi, j, l)=H_{B}(\xi, j)\left\{h_{L}(j,-l)-\int_{J-1 / 2}^{J+1 / 2} h_{L}(y,-l) d y\right\} .
$$

Then

$$
h_{1}(\xi, l)=\sum_{j=1}^{\infty} I_{6}(\xi, j, l)+\sum_{j=1}^{\infty} I_{7}(\xi, j, l) .
$$

Apply Lemma 2.3 and Proposition 3.4 and then recall (2.16). We then obtain that

$$
\left|I_{6}(\xi, j, l)\right| \leq 2 c_{16}\left(c_{6}+(1 / \pi)\right) j^{-2 / 3} l^{-5 / 3}\left\{|\xi|^{-1} I_{(0,2|\xi|]}(j)+j^{-1} I_{(2|\xi|, \infty)}(j)\right\} .
$$

The first term on the right-hand side of $(3.18)$ is dominated by $C|\xi|^{-2 / 3} l^{-5 / 3}$. The second term is negligible as $O\left(|\xi|^{-1} l^{-5 / 3}\right)$, if we show

$$
\left|h_{L}(j,-l)-\int_{j-1 / 2}^{j+1 / 2} h_{L}(y,-l) d y\right| \leq C j^{-4 / 3} l^{-5 / 3} .
$$

To prove (3.19), we apply the identity

$$
h_{L}(y,-l)=h_{B}^{2}(i y, l)+\int_{0}^{\infty} h_{B}^{2}(i y, \hat{y}) h_{L}(\hat{y},-l) d \hat{y} .
$$

Combining this relation and (2.33), the difference between $h_{L}(j,-l)$ and the integral of $h_{L}(y,-l)$ over $[j-1 / 2, j+1 / 2)$ with respect to $y$ is not greater than $c_{11} j^{-4 / 3} l^{-5 / 3}$ plus

$$
c_{11} \int_{0}^{\infty} j^{-1} \hat{y}^{-1}\left(j^{-1} \wedge \hat{y}^{-1}\right) h_{L}(\hat{y},-l) d \hat{y} .
$$

Apply (2.11) to see that (3.21) is bounded by a constant multiple of

$$
2 c_{2} j^{-1} l^{-5 / 3} \int_{0}^{\infty} \hat{y}^{-1 / 3}\left(j^{-1} \wedge \hat{y}^{-1}\right) d \hat{y} .
$$

Thus we have (3.19). From the definition of $h_{2}$, we obtain that

$$
\begin{aligned}
h_{2}(\xi,-l)= & -\int_{0}^{1 / 2} h_{B}(\xi, y) h_{L}(y,-l) d y \\
& +\int_{1 / 2}^{\infty}\left\{H_{B}(\xi,\langle y\rangle)-h_{B}(\xi, y)\right\} h_{L}(y,-l) d y .
\end{aligned}
$$

By (2.11), the first term on the right-hand side above is bounded in absolute value by $(4 / \pi) c_{2}|\xi|^{-1} l^{-5 / 3}$. Put

$$
\begin{aligned}
& I_{8}(\xi, y, l)=\left\{H_{B}(\xi,\langle y\rangle)-h_{B}(\xi, y)\right\}\left\{h_{L}(y,-l)-h_{L}(\langle y\rangle,-l)\right\} \\
& I_{9}(\xi, y, l)=\left\{H_{B}(\xi,\langle y\rangle)-h_{B}(\xi,\langle y\rangle)\right\} h_{L}(\langle y\rangle,-l)
\end{aligned}
$$


and

$$
I_{10}(\xi, y, l)=\left\{h_{B}(\xi,\langle y\rangle)-h_{B}(\xi, y)\right\} h_{L}(\langle y\rangle,-l)
$$

Then

$$
\left\{H_{B}(\xi,\langle y\rangle)-h_{B}(\xi, y)\right\} h_{L}(y,-l)=I_{8}(\xi, y, l)+I_{9}(\xi, y, l)+I_{10}(\xi, y, l) .
$$

By (2.26) and (3.20), we obtain

$$
\begin{aligned}
\left|h_{L}(y,-l)-h_{L}(\langle y\rangle,-l)\right| \leq & c_{8} l^{-2}(1 \vee \log l) \\
& \left.+\int_{0}^{1 / 2} \mid h_{B}^{2}(i y, \hat{y})-h_{B}^{2}(i<y\rangle, \hat{y}\right) \mid h_{L}(\hat{y},-l) d \hat{y} \\
& +c_{8} \int_{1 / 2}^{\infty} \hat{y}^{-2}(1 \vee \log \hat{y}) h_{L}(\hat{y},-l) d \hat{y} .
\end{aligned}
$$

Since $h_{B}^{2}(i y, \cdot)$ is a sub-probability density, the second term on the right-hand side above is at most $4 c_{2} l^{-5 / 3}$. By (2.11), the third term is bounded by a constant multiple of $l^{-5 / 3}$. Combining this and Lemma $2.3,\left|I_{8}(\xi, y, l)\right|$ is bounded by a constant multiple of $|\xi-y|^{-2} l^{-5 / 3}$. By (2.16), the integral of $|\xi-y|^{-2}$ over $[1 / 2, \infty)$ with respect to $y$ is negligible as $O\left(|\xi|^{-1}\right)$. By virtue of $(1.7)$ and (2.36), we have only to give a bound of

$$
\sum_{j=1}^{\infty}|\xi-j|^{-3} h_{L}(j,-l)
$$

instead of the integral of $\left|I_{9}(\xi, y, l)\right|$ and $\left|I_{10}(\xi, y, l)\right|$ over $[1 / 2, \infty)$ with respect to $y$. The difference between this sum and

$$
\int_{1 / 2}^{\infty}|\xi-\langle y\rangle|^{-3} h_{L}(y,-l) d y
$$

is negligible as $O\left(|\xi|^{-3} l^{-5 / 3}\right)$ in view of (3.19). By the same argument as showing that $(3.21)$ is $O\left(j^{-4 / 3} l^{-5 / 3}\right)$ and by employing the obvious inequality $|\xi-\langle y\rangle| \geq$ $(1 / 8)(|\xi| \vee y),(3.24)$ is negligible as $O\left(|\xi|^{-4 / 3} l^{-5 / 3}\right)$. Hence the desired estimate (1.2) has been verified for $\xi \in C^{*} \backslash L$ and $\mathfrak{R}(\xi) \leq \mathfrak{I}(\xi)$.

It remains to show that for $\xi \in C^{*} \backslash L$ and $\mathfrak{R}(\xi)>\mathfrak{I}(\xi)$,

$$
\begin{aligned}
& \left|H_{L}(\xi,-l)-h_{L}(\xi,-l)\right| \\
& \quad=\left|\sum_{j=1}^{\infty} H_{B}(i \bar{\xi}, j) H_{L}(i j,-l)-\int_{0}^{\infty} h_{B}(i \bar{\xi}, y) h_{L}(l y,-l) d y\right| \\
& \quad \leq C|\xi|^{-2 / 3} l^{-5 / 3} .
\end{aligned}
$$

The first relation holds for similar reason as (3.16). By using the bound

$$
\left|H_{L}(i j,-l)-h_{L}(i j,-l)\right| \leq \hat{C}^{\prime \prime} j^{-2 / 3} l^{-5 / 3}
$$


which has already been shown above, the same argument as in the proof of (3.17) verifies the last inequality. The proof of Theorem 1.1 is complete.

Proof of Theorem 1.2. We consider the case $\xi=k$. To estimate $H_{L}(k,-l)$, we will show that for $y>0$ and $b \geq k$,

$$
\left|\gamma \tilde{H}_{L}(y,-l)\right| \leq 2 \cdot 4{ }^{b} H_{L}(k,-l)+K(b) l^{-5 / 3},
$$

with a function $K(b)$ approaching zero as $b$ goes to infinity. Since $h_{L}(k, \cdot)$ is a probability density, $\left|h_{L} \gamma \tilde{H}_{L}(k,-l)\right|$ is also bounded by the right-hand side of (3.25). Noticing this and (3.25) and using (2.8), we obtain that

$$
\begin{aligned}
h_{L}(k,-l)-2 K(b) l^{-5 / 3} \leq & |\gamma(k,-l)|+\left|h_{L} \gamma(k,-l)\right| \\
& +\left(4^{b+1}+1\right) H_{L}(k,-l) .
\end{aligned}
$$

According to (2.31) and (3.6), the first and second terms on the right-hand side above are negligible as $O\left(l^{-11 / 6}\right)$. If $l$ is large enough, then (2.12) states that

$$
h_{L}(k,-l) \geq(1 / 2)^{5 / 3} c_{0} l^{-5 / 3} \text {. }
$$

Now if we choose $b$ such that

$$
K(b) \leq(1 / 3)(1 / 2)^{5 / 3} c_{0},
$$

then (1.3) has been verified for $\xi=k$.

For the proof of (3.25), we use the inequality

$$
\left|\gamma \tilde{H}_{L}(y,-l)\right| \leq \int_{1 / 2}^{b}|\gamma(y,-\tilde{y})| \tilde{H}_{L}(\tilde{y},-l) d \tilde{y}+\int_{b}^{\infty}|\gamma(y,-\tilde{y})| \tilde{H}_{L}(\tilde{y},-l) d \tilde{y} .
$$

If $k, j \in(0,\langle b\rangle] \cap N$, then the probability that random walk starting at $k$ reaches $j$ before visiting $L$ is at least $4^{-b}$. Hence

$$
H_{L}(j,-l) \leq 4{ }^{b} H_{L}(k,-l), \quad(j \in(0,\langle b\rangle] \cap N),
$$

and the first term on the right-hand side of (3.26) is at most $2 \cdot 4^{b} H_{L}(k,-l)$. The second term is bounded by

$$
\int_{b}^{\infty}|\gamma(y,-\tilde{y})|\left|H_{L}(\tilde{y},-l)-h_{L}(\tilde{y},-l)\right| d \tilde{y}+\int_{b}^{\infty}|\gamma(y,-\tilde{y})| h_{L}(\tilde{y},-l) d \tilde{y} .
$$

The first term of (3.27) is bounded by a constant multiple of $b^{-4 / 3}(1 \vee \log b) l^{-5 / 3}$ according to Lemma 2.4 and Proposition 3.3. Lemma 2.4 and (2.11) give

$$
|\gamma(y,-\tilde{y})| h_{L}(\tilde{y},-l) \leq 2 c_{2} c_{7} \tilde{y}^{-4 / 3}(1 \vee \log \tilde{y}) l^{-5 / 3} .
$$

Then the integral of $|\gamma(y,-\tilde{y})| h_{L}(\tilde{y},-l)$ over $[b, \infty)$ is bounded by a constant multiple of $b^{-1 / 3}(1 \vee \log b) l^{-5 / 3}$. Thus the second term on the right-hand side of (3.26) is at most

$$
C b^{-1 / 3}(1 \vee \log b) l^{-5 / 3}
$$


It is clear that this approaches zero as $b$ goes to infinity. This completes the proof of (3.25).

We consider the case $\xi \in C^{*} \backslash L$ and $\mathfrak{R}(\xi) \leq \mathfrak{I}(\xi)$. By combining (2.1) and the bound (1.3) for $\xi=1$ which has already been shown above, we obtain

$$
H_{L}(\xi,-l) \geq H_{B}(\xi, 1) H_{L}(1,-l) \geq H_{B}(\xi, 1) c_{1}(1) l^{-5 / 3} .
$$

Notice that $H_{B}(\xi, 1)>0$. Then (1.3) has been verified for this case. For $\xi \in C^{*} \backslash L$ with $\mathfrak{R}(\xi)>\mathfrak{I}(\xi),(2.2)$ and the bound that (1.3) for $\xi=i$ give the desired lower bound of $H_{L}(\xi,-l)$. The proof of Theorem 1.2 is complete.

Proof of Theorem 1.3. The proof is similar to that of Theorem 1.2. First we will show the following.

Lemma 3.6. For any $l \in N$, there exists $\hat{c}_{2}(l)$ such that for $k \in N$,

$$
H_{L}(k,-l) \geq \hat{c}_{2}(l) k^{-2 / 3} \text {. }
$$

Proof. By Lemma 2.4 and (3.1), $|\gamma(y,-l)|$ and $\left|\gamma h_{L}(y,-l)\right|$ are bounded by a constant multiple of $y^{-1}(1 \vee \log y)$. We will show that for $b \geq l$,

$$
\begin{aligned}
\int_{1 / 2}^{\infty} \tilde{H}_{L}(k,-y) y^{-1}(1 \vee \log y) d y \leq & 2 \cdot 4^{b+1} b(1 \vee \log b) H_{L}(k,-l) \\
& +\hat{K}(b) k^{-2 / 3}
\end{aligned}
$$

with a function $\hat{K}(b)$ approaching zero as $b$ goes to infinity. (3.28) implies that $|\tilde{H} \gamma(k,-l)|$ and $\left|\tilde{H} \gamma h_{L}(k,-l)\right|$ are bounded by a constant multiple of the righthand side of (3.28). Noticing this and using (2.9), we obtain that

$$
\begin{aligned}
h_{L}(k,-l)-2 C \hat{K}(b) k^{-2 / 3} \leq & |\gamma(k,-l)|+\left|\gamma h_{L}(k,-l)\right| \\
& +\left\{4^{b+2} b(1 \vee \log b) C+1\right\} H_{L}(k,-l) .
\end{aligned}
$$

If $k$ and $b$ are large enough, then the left-hand side above is at least $(1 / 3)$. $(1 / 2)^{5 / 3} c_{0} l^{-1 / 3} k^{-2 / 3}$. According to (2.31) and (3.1), the first and second terms on the right-hand side are negligible as $O\left(k^{-1}(1 \vee \log k)\right)$. The desired estimate follows.

For the proof of (3.28), we use the inequality

$$
\begin{aligned}
\int_{1 / 2}^{\infty} H_{L}(k,-y) y^{-1}(1 \vee \log y) d y \leq & 2 b(1 \vee \log b) \max _{1 \leq j \leq\langle b\rangle} H_{L}(k,-j) \\
& +\int_{b}^{\infty} \tilde{H}_{L}(k,-y) y^{-1}(1 \vee \log y) d y
\end{aligned}
$$

If $j, l \in(0,\langle b\rangle] \cap N$, then the probability that the random walk starting at $-j+i$ visits $L$ for the first time at $-l$ is at least $4^{-b-1}$. Hence

$$
H_{L}(k,-j) \leq 4^{b+1} H_{L}(k,-l) \quad(j \in(0,\langle b\rangle] \cap N),
$$


and the first term on the right-hand side of (3.29) is at most $2 \cdot 4^{b+1} b(1 \vee \log b)$. $H_{L}(k,-l)$. By Proposition 3.3, the difference between the second term and

$$
\int_{b}^{\infty} h_{L}(k,-y) y^{-1}(1 \vee \log y) d y
$$

is at most $3 c_{14} b^{-4 / 3}(1 \vee \log b) k^{-2 / 3}$. By (2.11) and the inequality $h(k,-y) \leq$ $k^{-2 / 3} y^{-1 / 3}$, the integral of $h(k,-y) y^{-1}(1 \vee \log y)$ over $[b,+\infty)$ is bounded by a constant multiple of $k^{-2 / 3} b^{-1 / 3}(1 \vee \log b)$. Thus the integral (3.30) is at most

We have (3.28).

$$
C b^{-1 / 3}(1 \vee \log b) k^{-2 / 3} \text {. }
$$

Let

$$
V^{*}(\delta)=\left\{\xi \in C^{*} \backslash L:-(\pi / 2)+\delta<\arg (\xi)<\pi-\delta\right\} .
$$

Next we consider the case $\xi \in V^{*}(\delta)$ and $\mathfrak{R}(\xi) \leq \mathfrak{I}(\xi)$. By (2.1) and Lemma 3.6, we obtain

$$
H_{L}(\xi,-l) \geq \sum_{|\xi| \leq J \leq 2|\xi|} H_{B}(\xi, j) H_{L}(j, l) \geq 2^{-2 / 3} \hat{c}_{2}(l)|\xi|^{-2 / 3}\left\{\sum_{|\xi| \leq J \leq 2|\xi|} H_{B}(\xi, j)\right\} .
$$

By the invariance principle if $|\xi|$ is large enough, then the last factor on the righthand side of the last inequality is at least a constant depending only on $\delta$. Thus we obtain that there exists a constant $c_{2}^{\prime}(l)$ such that for $\xi \in V^{*}(\delta)$ with $\mathfrak{R}(\xi) \leq$ $\mathfrak{I}(\xi)$

$$
H_{L}(\xi,-l) \geq c_{2}^{\prime}(l)|\xi|^{-2 / 3} \text {. }
$$

For $\xi \in V^{*}(\delta)$ with $\mathfrak{R}(\xi)>\mathfrak{I}(\xi)$, the desired lower bound follows from (2.2) and the above bound. The proof of Theorem 1.3 is complete.

Proof of Corollary 1.4. Combining the first inequality in (2.13) and Theorem 1.1, we can choose $n_{0}$ such that for $\xi \in V^{*}(\delta), l \in N$ with $|\xi|, l>n_{0}$,

$$
(1 / 5) h_{L}(\xi,-l) \leq h_{L}(\xi,-l)-4 C_{0}|\xi|^{-2 / 3} l^{-5 / 3} \leq H_{L}(\xi,-l),
$$

where $C_{0}$ is the same constant as in Theorem 1.1. The case when $|\xi| \leq n_{0}$ or $l \leq$ $n_{0}$ is disposed of by Theorem 1.2, 1.3 and the last inequality in (2.13). Thus we have the lower bound as required. The upper bound is immediate.

\section{Proof of Theorems $\mathbf{1 . 5}$ and $\mathbf{1 . 6}$}

In this section we prove Theorems 1.5 and 1.6, and then show (1.16), (1.19) in Remark 1.3 and the first assertion in Remark 1.4. To begin with, we consider

$$
\begin{aligned}
P(k, l) & :=P_{k}\left\{S\left(\tau_{L}\right) \in\{-l,-i l\}\right\} \\
& =P_{i k}\left\{S\left(\tau_{L}\right) \in\{-l,-i l\}\right\} \\
& =H_{L}(k,-l)+H_{L}(i k,-l) \quad(l \in \boldsymbol{N})
\end{aligned}
$$

and show the following. 
Proposition 4.1. For each $k \in N$, the following limit exists

$$
\hat{c}^{*}(k):=\lim _{l \rightarrow \infty} l^{5 / 3} P(k, l) .
$$

The function $\hat{c}^{*}$ is characterized as a unique solution to (1.13) with the boundary condition $\lim _{k \rightarrow \infty} k^{-2 / 3} \hat{c}^{*}(k)=2 c_{0}$, where $c_{0}$ is the same constant as in Theorem 1.5 .

Proposition 4.2. For any $l \in N$, the following limit exists

$$
\hat{c}_{*}(l):=\lim _{k \rightarrow \infty} k^{2 / 3} P(k, l) .
$$

The function $\hat{c}_{*}$ is characterized as a unique solution to (1.14) with the boundary condition $\lim _{l \rightarrow \infty} l^{1 / 3} \hat{c}_{*}(l)=2 c_{0}$, where $c_{0}$ is the same constant as in Theorem 1.5.

Proof of Proposition 4.1. We have shown that for $k, l \in N$,

$$
0<c_{1}(k)+c_{1}(i k) \leq l^{5 / 3} P(k, l) \leq 4 c c_{2} k^{2 / 3} .
$$

In fact, the first two inequalities follow from Theorem 1.2 and the last inequality follows from Corollary 1.4 and (2.11). For fixed $k, l^{5 / 3} P(k, l)$ is bounded. By Cantor's diagonal argument, there exists a sequence $\left\{l_{n}\right\}$ such that $l_{n}^{5 / 3} P\left(k, l_{n}\right)$ is convergent for all $k \in N$. We write

$$
\tilde{c}(k):=\lim _{n \rightarrow \infty} l_{n}^{5 / 3} P\left(k, l_{n}\right) .
$$

The function $\tilde{c}$ satisfies the following condition

(a) $\tilde{c}(k)>0(k \in N)$

(b) $\sup _{k \in N} k^{-2 / 3} \tilde{c}(k) \leq 4 c c_{2}$,

(c) $\lim _{k \rightarrow \infty} k^{-2 / 3} \tilde{c}(k)=2 c_{0}$.

(a) and (b) follow from (4.2). We notice that $l^{5 / 3}\left(h_{L}(k,-l)+h_{L}(i k,-l)\right)$ approaches $2 c_{0} k^{2 / 3}$ as $l \rightarrow \infty$. This and Theorem 1.1 imply (c).

Let us show that $\tilde{c}$ satisfies (1.13). In view of (4.1), the strong Markov property gives

$$
P(k, l)=H_{B}(i k,-l)+\sum_{j=1}^{\infty} H_{B}(i k, j) P(j, l) .
$$

We multiply the both sides by $l^{5 / 3}$ and take limit along $l_{n}$. We use the last inequality in (4.2) to justify an interchange of the order of limit and summation. By the dominated convergence theorem, we conclude that $\tilde{c}$ satisfies (1.13). result.

Proof of Proposition 4.1 is complete if we prove the following uniqueness

Lemma 4.3. Suppose that $\tilde{c}_{1}$ is a solution to (1.13) and satisfies (c). Suppose $\tilde{c}_{2}$ is a solution to (1.13) and satisfies (a) and (c). Then $\tilde{c}_{1}=\tilde{c}_{2}$. 
Proof. By (c), $\tilde{c}_{1}(k) / \tilde{c}_{2}(k)$ tends to 1 as $k \rightarrow \infty$. Suppose that there exists $k_{0}$ such that $\tilde{c}_{1}\left(k_{0}\right) / \tilde{c}_{2}\left(k_{0}\right) \neq 1$. By symmetry, we may assume $\tilde{c}_{1}\left(k_{0}\right) / \tilde{c}_{2}\left(k_{0}\right)>1$, so that the function $\tilde{c}_{1}(k) / \tilde{c}_{2}(k)$ attains the maximum at some point, $k^{*}$ say. Since $\tilde{c}_{1}$ and $\tilde{c}_{2}$ are solutions to (1.13), we obtain

$$
\sum_{j=1}^{\infty} H_{B}\left(i k^{*}, j\right)\left\{\tilde{c}_{1}(j)-\frac{\tilde{c}_{1}\left(k^{*}\right)}{\tilde{c}_{2}\left(k^{*}\right)} \tilde{c}_{2}(j)\right\}=0 .
$$

According to the manner of the choice of $k^{*}, \tilde{c}_{1}(j)-\left(\tilde{c}_{1}\left(k^{*}\right) / \tilde{c}_{2}\left(k^{*}\right)\right) \tilde{c}_{2}(j) \leq 0$. Hence

in particular

$$
\tilde{c}_{1}(j)-\frac{\tilde{c}_{1}\left(k^{*}\right)}{\tilde{c}_{2}\left(k^{*}\right)} \tilde{c}_{2}(j)=0 \quad(j \in \mathbf{N})
$$

$$
\frac{\tilde{c}_{1}\left(k^{*}\right)}{\tilde{c}_{2}\left(k^{*}\right)}=\lim _{J \rightarrow \infty} \frac{\tilde{c}_{1}(j)}{\tilde{c}_{2}(j)}=1 .
$$

This contradicts our supposition that $\tilde{c}_{1}\left(k^{*}\right) / \tilde{c}_{2}\left(k^{*}\right)>1$.

Proof of Proposition 4.2. We can prove Proposition 4.2 in a way analogous to the proof of Proposition 4.1. The proof is only outlined. There exists a sequence $\left\{k_{n}\right\} \subset N$ such that for every $l \in N, k_{n}^{2 / 3} P\left(k_{n}, l\right)$ converges. Let $\bar{c}(l)$ be the limit. Then $\bar{c}(l)$ satisfies

and

$$
\begin{aligned}
& \left(\mathrm{a}^{\prime}\right) \bar{c}(l)>0(l \in N), \\
& \left(\mathrm{b}^{\prime}\right) \sup _{l \in N} l^{1 / 3} \bar{c}(l) \leq 4 c c_{2}
\end{aligned}
$$

(c') $\lim _{l \rightarrow \infty} l^{1 / 3} \bar{c}(l)=2 c_{0}$. $k, l \in \boldsymbol{N}$,

Let us show that $\bar{c}$ satisfies (1.14). By iterating (4.3), we obtain that for

$$
P(k, l)=\sum_{n=1}^{\infty} H_{B}^{n}(i k, l) .
$$

From this, it is easy to see that

$$
P(k, l)=H_{B}(i k, l)+\sum_{j=1}^{\infty} P(k, j) H_{B}(i j, l) .
$$

Noticing that $k^{2 / 3} H_{B}(i k, l)$ vanish as $k \rightarrow \infty$, we observe that it is enough to show that

$$
\lim _{n \rightarrow \infty} k_{n}^{2 / 3} \sum_{j=1}^{\infty} P\left(k_{n}, j\right) H_{B}(i j, l)=\sum_{j=1}^{\infty} \bar{c}(j) H_{B}(i j, l) .
$$

This is immediate from the inequality

$$
k^{2 / 3} P(k, j) \leq C j^{-1 / 3}
$$


that follows from Corollary 1.4 and (2.11). Thus $\bar{c}$ satisfies (1.14). As before Proposition 4.2 now follows from the following uniqueness result proved as Lemma 4.3.

Lemma 4.4. Suppose that $\bar{c}_{1}$ is a solution to (1.14) and satisfies $\left(\mathrm{c}^{\prime}\right)$. Suppose $\bar{c}_{2}$ is a solution to (1.14) and satisfies $\left(\mathrm{a}^{\prime}\right)$ and $\left(\mathrm{c}^{\prime}\right)$. Then $\bar{c}_{1}=\bar{c}_{2}$.

Lemma 4.5. There exists a constant $C$ such that for $k, l \in N$,

$$
H_{B}^{2}(i k, l) \leq C k\left(k^{-2} \wedge l^{-2}\right)(1 \vee \log [(k \vee l) /(k \wedge l)]) .
$$

Proof. In view of (2.30),

$$
h_{B}^{2}(i k, l)= \begin{cases}\frac{1}{(k+l) \pi^{2}} \frac{\log (k / l)}{1-(l / k)} & (k \neq l) \\ \frac{1}{2 k \pi^{2}} & (k=l) .\end{cases}
$$

By (2.31), the difference between $H_{B}^{2}(i k, l)$ and $h_{B}^{2}(i k, l)$ is negligible as $O\left(k^{-1} l^{-2}\right)$. The proof of Lemma 4.5 is complete.

Proof of Theorem 1.5. The same argument as in the proof of Proposition 4.1 shows that there exists $c^{*}(k):=\lim _{l \rightarrow \infty} l^{5 / 3} H_{L}(k,-l)$, $c^{*}$ satisfies $c^{*}(k)>0$ $(k \in \boldsymbol{N})$ and $c^{*}$ is a solution to

$$
c^{*}(k)=\sum_{j=1}^{\infty} H_{B}^{2}(i k, j) c^{*}(j)
$$

with the boundary condition $\lim _{k \rightarrow \infty} k^{-2 / 3} c^{*}(k)=c_{0}$. Here we have applied Lemma 4.5 and (2.3). Uniqueness of a solution to (4.6) which satisfies the boundary condition $\lim _{k \rightarrow \infty} k^{-2 / 3} c^{*}(k)=c_{0}$ is verified as in the proof of Lemma 4.3.

We must show that $(1 / 2) \hat{c}^{*}=c^{*}$. Since $\hat{c}^{*}$ is a solution to $(1.13), \hat{c}^{*}$ is also a solution to (4.6). Moreover $\hat{c}^{*}$ satisfies (a) and $\lim _{k \rightarrow \infty} k^{-2 / 3} \hat{c}^{*}(k)=2 c_{0}$. Hence by uniqueness $(1 / 2) \hat{c}^{*}=c^{*}$.

Proof of Theorem 1.6. The proof of the first and second assertions is similar to that of Theorem 1.5. The proof of the last assertion is based on the relation

$$
H_{L}(k, 0)=H_{B}(i k, 0)+\sum_{j=1}^{\infty} P(k, j) H_{B}(i j, 0)
$$

that follows from (4.4) and $H_{L}(k, 0)=\sum_{n=1}^{\infty} H_{B}^{n}(i k, 0) . \quad((4.4)$ is also valid for $l=0)$. The same reason as showing that $\bar{c}$ satisfies (1.14) implies that $\lim _{k \rightarrow \infty} k^{2 / 3} H_{L}(k, 0)$ exists and the limit is expressed as (1.15). The proof of Theorem 1.6 is complete. 
Proofs of (1.16) and (1.19). The same arguments as showing that $\tilde{c}$ satisfies (1.13) deduce (1.16) from (2.1) and (2.2). If $|\xi| \geq 2 l$ and $(\pi / 8)<\arg (\xi)<\pi$, then (2.1) and Lemma 2.3 imply that

$$
H_{L}(\xi,-l)=\sum_{j=1}^{\infty} h_{B}(\xi, j) H_{L}(j,-l)+O\left(|\xi|^{-1}\right) .
$$

We rewrite $h_{B}(\xi, j)$ in the form

$$
h_{B}(\xi, j)=|\xi|^{-5 / 3} h_{B}\left(\frac{\xi}{|\xi|}, \frac{j}{|\xi|}\right)\left(\frac{j}{|\xi|}\right)^{-2 / 3} j^{2 / 3} .
$$

By this and Theorem 1.6, $|\xi|^{2 / 3} H_{L}(\xi,-l)$ tends to the right-hand side of (1.19) as $|\xi|$ goes to infinity in such a way $\arg (\xi) \rightarrow \theta$, showing (1.19).

Let us verify that both $k^{-2 / 3} c^{*}(k)$ and $l^{1 / 3} c_{*}(l)$ are non-constant functions. We decompose $H_{B}(i m, j)$ according to the value of $S(1)$ to obtain that for $m \in N$,

$$
\begin{aligned}
H_{B}(i m, j)= & \frac{1}{4}\left\{H_{B}(i(m+1), j)+H_{B}(1+i m, j)\right. \\
& \left.+H_{B}(-1+i m, j)+H_{B}(i(m-1), j)\right\},
\end{aligned}
$$

where $H_{B}(0, j)=\delta(0, j)$. Multiply (4.8) by $c^{*}(j)$ and make summation over $j \in N$. Further, take $m=1$ and use (1.13). Then

$$
c^{*}(1)=\frac{1}{4}\left\{c^{*}(2)+\sum_{j=1}^{\infty} H_{B}(1+i, j) c^{*}(j)+\sum_{j=1}^{\infty} H_{B}(-1+i, j) c^{*}(j)\right\} .
$$

Assume that $k^{-2 / 3} c^{*}(k)$ is constant. Then, from (1.13) it follows that $k^{2 / 3}=$ $\sum_{j=1}^{\infty} H_{B}(i k, j) j^{2 / 3}$ and, since $H_{B}(m+i, j)=H_{B}(i, j-m)$, the previous equality yields

$$
H_{B}(i, 0)=4-2^{2 / 3}-\sum_{j=1}^{\infty} H_{B}(i, j)\left\{(j+1)^{2 / 3}+(j-1)^{2 / 3}\right\} .
$$

The infinite sum on the right-hand side above is at most 2 since $x^{2 / 3}$ is concave, i.e., $(j-1)^{2 / 3}+(j+1)^{2 / 3} \leq 2 j^{2 / 3}$. On the other hand, $H_{B}(i, 0)$ is expressed as

$$
H_{B}(i, 0)=(1 / 4) G_{B}(i, i)=(1 / 4) a(2 i)
$$

by virtue of (1.4) and (1.5). The values of $a(\xi)$ are computed in Spitzer [8, p. 149], according to which we obtain that

$$
2-2^{2 / 3} \leq(1 / 4) a(2 i)=1-(2 / \pi) .
$$

But in fact $2-2^{2 / 3}>1-(2 / \pi)$. Thus we may conclude that $k^{-2 / 3} c^{*}(k)$ is not constant.

Multiply (4.8) by $c_{*}(m)$ and make summation over $m \in N$. Further, take $j=2$ and use (1.14). Then we obtain that 
$c_{*}(2)=\frac{1}{4}\left\{c_{*}(1)+c_{*}(3)+\sum_{m=1}^{\infty} c_{*}(m) H_{B}(i(m+1), 2)+\sum_{m=2}^{\infty} c_{*}(m) H_{B}(i(m-1), 2)\right\}$.

If $l^{1 / 3} c_{*}(l)$ is a constant function, then this equality yields

$$
\begin{aligned}
\left(2-2^{-1 / 3}\right) H_{B}(i, 2)= & -4 \cdot 2^{-1 / 3}+1+3^{-1 / 3}+2 H_{B}(i, 2) \\
& +\sum_{m=2}^{\infty}\left\{(m-1)^{-1 / 3}+(m+1)^{-1 / 3}\right\} H_{B}(i m, 2)
\end{aligned}
$$

and from (1.14) it follows that $l^{-1 / 3}=\sum_{m=1}^{\infty} m^{-1 / 3} H_{B}(i m, l)$. The last term on the right-hand side above is at least $2 \sum_{m=2}^{\infty} m^{-1 / 3} H_{B}(i m, 2)$ since $x^{-1 / 3}$ is convex, i.e., $(m-1)^{-1 / 3}+(m+1)^{-1 / 3} \geq 2 m^{-1 / 3}$. On the other hand, $H_{B}(i, 2)=$ $(1 / 4)\{a(-2+2 i)-a(-2)\}=(10 / 3 \pi)-1$. Thus

$$
\left(2-2^{-1 / 3}\right)\left(\frac{10}{3 \pi}-1\right) \geq 1+3^{-1 / 3}-2^{2 / 3} \text {. }
$$

This contradicts the actual inequality $\left(2-2^{-1 / 3}\right)(10 / 3 \pi-1)<1+3^{-1 / 3}-2^{2 / 3}$. Thus we also conclude that $l^{1 / 3} c_{*}(l)$ is not constant.

\section{Proof of Theorem 1.7}

In this section we state some known results and then prove Theorem 1.7.

Lemma 5.1 (Lawler [7, Theorem 2.1.3]). There exists a constant $C$ and $a$ natural number $k_{0}$ such that if $A$ is a subset of $C^{*}$ that is contained in the ball of radius $n$ and $|\xi| \geq k_{0} n$, then

$$
\left|H_{A}(\xi, \zeta)-\mu_{A}(\zeta)\right| \leq C \frac{n}{|\xi|}\left(\log \frac{|\xi|}{n}\right) \mu_{A}(\zeta), \quad(\zeta \in A)
$$

Lemma 5.2 (Kesten [4]). Let $U(n)=\left\{-l \in C^{*}: 0 \leq l \leq n\right\}$. Then there exists a constant $C$ such that for $-l \in U(n)$,

$$
\mu_{U(n)}(-l) \leq C\left((n-l+1)^{-1 / 2} \vee(l+1)^{-1 / 2}\right) n^{-1 / 2} .
$$

The next lemma is deduced from Theorem 1.1 by an easy modification of Lawler's proof of Proposition 2.4.10 in Lawler [7].

LEMMA 5.3. There exists a constant $C$ such that

$$
\mu_{L(n)}(-l) \leq \begin{cases}C(l+1)^{-1 / 3} n^{-2 / 3}, & (0 \leq l<n / 2), \\ C(n-l+1)^{-1 / 2} n^{-1 / 2}, & (n / 2 \leq l \leq n) .\end{cases}
$$

Proof. If $l \in[n / 5, n] \cap N$, then the bound of Lemma 5.3 is immediate from Lemma 5.2. By virtue of (1.22), it therefore suffices to show that for $l \in$ 
$[0, n / 5) \cap(\{0\} \cup \boldsymbol{N})$,

$$
P_{-l}\left\{\tau_{\partial C(2 n)}<\tau_{L(n)}\right\} \leq C(l+1)^{-1 / 3} n^{-2 / 3},
$$

where $C(r)=\left\{\xi \in C^{*}:|\xi| \leq r\right\}$ and $\partial C(r)=\left\{\xi \in C^{*} \backslash C(r)|\exists \eta \in C(r):| \xi-\eta \mid=\right.$ $1\}$ as in (1.1). Let us extend the function $P(\cdot, l)$ to the one on $C^{*} \backslash L$ by

$$
P(\xi, l)=H_{L}(\xi,-l)+H_{L}(i \bar{\xi},-l) \text {. }
$$

The strong Markov property shows that for $n \geq 2$ and $\xi \in C(n / 2) \backslash L$,

$$
\sum_{j=n+1}^{\infty} P(\xi, j)=\sum_{\eta \in \partial C(n) \backslash L} P_{\xi}\left\{\tau_{\partial C(n)}<\tau_{L}, S\left(\tau_{\partial C(n)}\right)=\eta\right\}\left(\sum_{J=n+1}^{\infty} P(\eta, j)\right) .
$$

Theorem 1.1 and (2.14) imply that for $j>2|\xi|$,

$$
P(\xi, j) \leq 2\left(c_{5}+9 C_{0}\right)|\xi|^{2 / 3} j^{-5 / 3} \text {. }
$$

Thus the left-hand side of (5.2) is bounded by a constant multiple of $|\xi|^{2 / 3} n^{-2 / 3}$. On the other hand, the invariance principle shows that there exists a positive constant $C$ such that for $n \in N$ and $\eta \in \partial C(n) \backslash L$,

$$
\sum_{j=n+1}^{\infty} P(\eta, j) \geq C
$$

Hence for $\xi \in C(n / 2) \backslash L$,

$$
P_{\xi}\left\{\tau_{\partial C(n)}<\tau_{L(n)}\right\}=P_{\xi}\left\{\tau_{\partial C(n)}<\tau_{L}\right\} \leq c_{19}|\xi|^{2 / 3} n^{-2 / 3} .
$$

Since

$$
\begin{aligned}
P_{0}\left\{\tau_{\partial C(2 n)}<\tau_{L(n)}\right\} & \leq(1 / 4) P_{i}\left\{\tau_{\partial C(n)}<\tau_{L(n)}\right\}+(1 / 4) P_{1}\left\{\tau_{\partial C(n)}<\tau_{L(n)}\right\} \\
& =(1 / 2) P_{i}\left\{\tau_{\partial C(n)}<\tau_{L(n)}\right\}
\end{aligned}
$$

(5.1) is shown to hold for $l=0$. Corollary 1.4 and (2.13) imply that

$$
\sum_{j=n+1}^{\infty} P(i, j) \geq c_{20} n^{-2 / 3}
$$

where $c_{20}$ is a positive constant. It is clear that the second factor on the righthand side of (5.2) is at most 1. Combining this and (5.2), we also have

$$
P_{i}\left\{\tau_{\partial C(n)}<\tau_{L(n)}\right\} \geq c_{20} n^{-2 / 3} .
$$

Assume $l \in[1, n / 5) \cap N$. To get the upper bound of $P_{-l}\left\{\tau_{\partial C(n)}<\tau_{L(n)}\right\}$, we use the following inequality;

$$
P_{-l}\left\{\tau_{\partial C(n)}<\tau_{L(n)}\right\} \leq P_{-l}\left\{\tau_{\partial C(2 l)}<\tau_{L(n)}\right\}\left(\sup _{\xi \in \partial C(2 l) \backslash L(n)} P_{\xi}\left\{\tau_{\partial C(n)}<\tau_{L(n)}\right\}\right) .
$$


Replacing $\tau_{\partial C(2 l)}$ by $\tau_{-l+\partial C(l)}$ and using the spatial homogeneity, we see that the first factor on the right-hand side of the above inequality is at most

$$
P_{0}\left\{\tau_{\partial C(l)}<\tau_{U}\right\} \leq c_{21} l^{-1}
$$

where $U:=\{\xi \in C: \mathfrak{I}(\xi)=0\}$ and the inequality above is shown in Lawler [7, §2.4]. By (5.4), the supremum of $P_{\xi}\left\{\tau_{\partial C(n)}<\tau_{L(n)}\right\}$ over $\xi \in \partial C(2 l) \backslash L(n)$ is at most $2^{2 / 3} c_{19} l^{2 / 3} n^{-2 / 3}$. Therefore, we have

$$
P_{-l}\left\{\tau_{\partial C(2 n)}<\tau_{L(n)}\right\} \leq P_{-l}\left\{\tau_{\partial C(n)}<\tau_{L(n)}\right\} \leq 2^{2 / 3} c_{19} c_{21} l^{-1 / 3} n^{-2 / 3} .
$$

This is the desired bound. The proof of Lemma 5.3 is complete.

To get the lower bound of $P_{-1}\left\{\tau_{\partial C(2 n)}<\tau_{L(n)}\right\}$, we replace $\tau_{L(n)}$ by $\tau_{L(2 n)}$ and use the fact that the probability that random walk starting at -1 reaches $i$ before visiting $\partial C(2 n) \cup L(n)$ is at least $1 / 16$. Then

$$
P_{-1}\left\{\tau_{\partial C(2 n)}<\tau_{L(n)}\right\} \geq \frac{1}{16} P_{i}\left\{\tau_{\partial C(2 n)}<\tau_{L(2 n)}\right\} .
$$

Combining this, (1.22) and (5.5), we also have the following lemma.

Lemma 5.4. There exists a positive constant $C$ such that

$$
\mu_{L(n)}(-1) \geq \mathrm{Cn}^{-2 / 3} \text {. }
$$

LEMMA 5.5.

$$
\lim _{n \rightarrow \infty} \sup _{\xi \in \partial C(n) \backslash L}\left|n^{2 / 3} H_{L}(\xi,-l)-\int_{0}^{\infty} h_{B}\left(\frac{\hat{\xi}}{|\xi|}, u\right) u^{-2 / 3} d u c_{*}(l)\right|=0,
$$

where

$$
\hat{\xi}= \begin{cases}\xi & \text { if } \mathfrak{R}(\xi) \leq \mathfrak{I}(\xi) \\ i \bar{\xi} & \text { if } \quad \mathfrak{R}(\xi)>\mathfrak{I}(\xi)\end{cases}
$$

Proof. The proof is similar to that of (1.19). The same arguments as showing (4.7) and (2.2) show that for $\xi \in \partial C(n) \backslash L$ with $\mathfrak{R}(\xi)>\mathfrak{I}(\xi)$,

$$
H_{L}(\xi,-l)=\sum_{j=1}^{\infty} h_{B}(i \bar{\xi}, j) H_{L}(i j,-l)+O\left(|\xi|^{-8 / 3}\right) .
$$

We notice that $\mathfrak{R}(i \bar{\xi})<\mathfrak{I}(i \bar{\xi})$ for $\mathfrak{R}(\xi)>\mathfrak{I}(\xi)$ and $\lim _{j \rightarrow \infty} j^{2 / 3} H_{L}(j,-l)=$ $\lim _{j \rightarrow \infty} j^{2 / 3} H_{L}(i j,-l)$ (see $\left.(1.20)\right)$. These reduce our problem to showing that uniformly for $\xi \in \partial C(n) \backslash L$ with $\mathfrak{R}(\xi) \leq \mathfrak{I}(\xi)$ and for $s=0,1$,

$$
|\xi|^{2 / 3} \sum_{j=1}^{\infty} h_{B}(\xi, j) H_{L}\left(i^{s} j,-l\right)-\int_{0}^{\infty} h_{B}\left(\frac{\xi}{|\xi|}, u\right) u^{-2 / 3} d u c_{*}(l) \rightarrow 0
$$

as $n \rightarrow+\infty$. The required uniformity comes from (2.16). 
Let $[s]$ be the greatest integer not exceeding $s$ and let

$$
P_{n}(\xi,-l)=P_{\xi}\left\{S\left(\tau_{L(n)}\right)=-l\right\} \text {. }
$$

Lemma 5.6. Let $q \in(0,1)$ be fixed. Then there exists a constant $C$ such that for $\xi \in \partial C\left(\left[n^{q}\right]\right) \backslash L$ and $l \in\left(0,\left[n^{q}\right] / 4\right] \cap N$,

$$
\left|P_{n}(\xi,-l)-H_{L}(\xi,-l)\right| \leq C n^{-2 / 3}
$$

and for $\xi \in\left(\partial C\left(\left[n^{q}\right]\right) \backslash L(n)\right) \cap L$ and $l \in\left(0,\left[n^{q}\right] / 4\right] \cap N$,

$$
P_{n}(\xi,-l) \leq C\left(n^{-q} \vee n^{-1+q / 3}\right) .
$$

Moreover, there exists a constant $C$ such that

$$
P_{n^{2}}\left\{\tau_{\partial C\left(\left[n^{q}\right]\right)}<\tau_{L(n)}\right\} \leq C n^{-2(1-q) / 3} .
$$

Proof. Let $\xi \in \partial C\left(\left[n^{q}\right]\right) \backslash L$ and $l \in\left(0,\left[n^{q}\right] / 4\right] \cap N$ and put

$$
\hat{L}=\{-l: l=0,1,2, \ldots\} \cup\{-i l: l=0,1,2, \ldots\} .
$$

By the strong Markov property, the difference $P_{n}(\xi,-l)-H_{L}(\xi,-l)$ is equal to the sum of $H_{L}(\xi, \eta) P_{n}(\eta,-l)$ over $\eta \in \hat{L} \backslash L(n)$. Therefore

$$
P_{n}(\xi,-l)-H_{L}(\xi,-l) \leq P_{\xi}\left\{S\left(\tau_{L}\right) \in \hat{L} \backslash L(n)\right\}\left(\sup _{\eta \in \hat{L} \backslash L(n)} P_{n}(\eta,-l)\right)
$$

We replace $\tau_{L(n)}$ by $\tau_{L\left(\left[n^{q}\right]\right)}$ and combine Lemmas 5.1 and 5.3. If $n$ is large enough, then the second factor on the right-hand side above is $O\left(n^{-2 q / 3}\right)$. We rewrite the first factor as

$$
P_{\xi}\left\{S\left(\tau_{L}\right) \in \hat{L} \backslash L(n)\right\}=\sum_{j=n+1}^{\infty} P(\xi, j) .
$$

If $n \geq 2|\xi|$, then the bound (5.3) implies that the right-hand side above is $O\left(n^{-2(1-q) / 3}\right)$. Thus we have (5.6).

The proof of (5.7) is similar to that of (5.6). Assume $\xi \in\left(\partial C\left(\left[n^{q}\right]\right) \backslash L(n)\right) \cap$ $L$ and $l \in\left(0,\left[n^{q}\right] / 4\right] \cap N$. To estimate $P_{n}(\xi,-l)$, we use the following inequality

$$
P_{n}(\xi,-l) \leq P_{\xi}\left\{S\left(\tau_{\hat{L}}\right)=-l\right\}+P_{\xi}\left\{S\left(\tau_{\hat{L}}\right) \in \hat{L} \backslash L(n)\right\}\left(\sup _{\eta \in \hat{L} \backslash L(n)} P_{n}(\eta,-l)\right) .
$$

By symmetry of random walk, we obtain that for $\xi \in C^{*}$ with $\mathfrak{R}(\xi)<0$, $\mathfrak{I}(\xi)<0$,

$$
P_{\xi}\left\{S\left(\tau_{\hat{L}}\right)=-j\right\} \leq H_{B}(-\xi, j) \quad \text { and } \quad P_{\xi}\left\{S\left(\tau_{\hat{L}}\right)=-\imath j\right\} \leq H_{B}(-i \bar{\xi}, j) .
$$

By (5.10) and Lemma 2.3, the first term on the right-hand side of (5.9) is at most $8\left(c_{6}+(1 / \pi)\right) n^{-q}$. If $j \geq 2|\xi|$, then $|\xi+j| \wedge|i \bar{\xi}+j| \geq j / 2$. Combining this inequality, (5.10) and Lemma 2.3, the first factor of the second term is 
bounded by $8\left(c_{6}+(1 / \pi)\right)|\xi| \sum_{\jmath=n+1}^{\infty} j^{-2}$. It has already been shown in the proof of (5.6) that the second factor of the second term is $O\left(n^{-2 q / 3}\right)$. The proof of (5.7) is complete.

If $n \geq 3$, then every random walk path from $n^{2}$ to $L\left(\left[n^{q}\right]\right)$ crosses $\partial C\left(\left[n^{q}\right]\right)$. By decomposing $P_{n^{2}}\left\{S\left(\tau_{L(n)}\right) \in L\left(\left[n^{q}\right]\right)\right\}$ according to the first crossing point, we obtain that for $n \geq 3$,

$$
\begin{aligned}
P_{n^{2}}\left\{S\left(\tau_{L(n)}\right) \in L\left(\left[n^{q}\right]\right)\right\} \geq & P_{n^{2}}\left\{\tau_{\partial C\left(\left[n^{q}\right]\right)}<\tau_{L(n)}\right\} \\
& \cdot\left(\inf _{\xi \in \partial C\left(\left[n^{q}\right]\right) \backslash L(n)} P_{\xi}\left\{S\left(\tau_{L(n)}\right) \in L\left(\left[n^{q}\right]\right)\right\}\right)
\end{aligned}
$$

If $\left[n^{q}\right]<n / 4$ and $n$ is large enough, then Lemmas 5.1 and 5.3 imply that the lefthand side of $(5.11)$ is bounded by a constant multiple of

$$
n^{-2 / 3} \sum_{j=0}^{\left[n^{q}\right]}(J+1)^{-1 / 3} .
$$

The sum is $O\left(n^{-2(1-q) / 3}\right)$. By making use of invariance principle, it is easy to show that there exists a positive constant $c_{22}$ (independent of $n$ ) such that for $\xi \in \partial C\left(\left[n^{q}\right]\right) \backslash L(n)$

$$
P_{\xi}\left\{S\left(\tau_{\hat{L}}\right) \in L\left(\left[n^{q}\right]\right)\right\} \geq c_{22} .
$$

Hence the second factor on the right-hand side of (5.11) is at least $c_{22}$ and we have (5.8).

Proof of Theorem 1.7. Let $q \in(0,1)$ be fixed. The proof is based on

$$
\begin{aligned}
P_{n}\left(n^{2},-l\right)= & \sum_{\xi \in \partial C\left(\left[n^{q}\right]\right) \backslash L(n)} P_{n^{2}}\left\{\tau_{\partial C\left(\left[n^{q}\right]\right)}<\tau_{L(n)}, S\left(\tau_{\partial C\left(\left[n^{q}\right]\right)}\right)=\xi\right\} \\
& \cdot P_{n}(\xi,-l), \quad\left(n \geq 3, l \in\left(0,\left[n^{q}\right] / 4\right] \cap N\right) .
\end{aligned}
$$

Since Kesten [5] provided the existence of $\lim _{n \rightarrow \infty} n^{2 / 3} \mu_{L(n)}(-l)$, Lemma 5.1 implies that there exists $\lim _{n \rightarrow \infty} n^{2 / 3} P_{n}\left(n^{2},-l\right)$ and the limit is equal to $\lim _{n \rightarrow \infty} n^{2 / 3} \mu_{L(n)}(-l)$. From Lemmas 5.5 and 5.6, the difference between the right-hand side of (5.12) and

$$
\begin{gathered}
n^{-2 q / 3} c_{*}(l) \sum_{\xi \in \partial C\left(\left[n^{q}\right]\right) \backslash L} P_{n^{2}}\left\{\tau_{\partial C\left(\left[n^{q}\right]\right)}<\tau_{L(n)}, S\left(\tau_{\partial C\left(\left[n^{q}\right]\right)}\right)=\xi\right\} \\
\cdot \int_{0}^{\infty} h_{B}\left(\frac{\hat{\xi}}{|\xi|}, u\right) u^{-2 / 3} d u
\end{gathered}
$$

is $o\left(n^{-2 / 3}\right)$. By (5.12) we conclude that

$$
\lim _{n \rightarrow \infty} n^{2 / 3} \mu_{L(n)}(-l)=\alpha c_{*}(l)
$$


where

$$
\begin{aligned}
\alpha= & \lim _{n \rightarrow \infty} n^{2(1-q) / 3} \sum_{\xi \in \partial C\left(\left[n^{q}\right]\right) \backslash L} P_{n^{2}}\left\{\tau_{\partial C\left(\left[n^{q}\right]\right)}<\tau_{L(n)}, S\left(\tau_{\partial C\left(\left[n^{q}\right]\right)}\right)=\xi\right\} \\
& \cdot \int_{0}^{\infty} h_{B}\left(\frac{\hat{\xi}}{|\xi|}, u\right) u^{-2 / 3} d u .
\end{aligned}
$$

It is immediate from Lemma 5.4 that $\alpha$ is a positive constant. The proof of Theorem 1.7 is complete.

\section{Proof of Theorem 1.8}

In this section we will prove Theorem 1.8.

Let

$$
\begin{aligned}
& U_{1}=\{\zeta \in C: \mathfrak{R}(\zeta)>0\} \cup\{\zeta \in C \backslash\{0\}:-\mathfrak{I}(\zeta) \leq \mathfrak{R}(\zeta) \leq 0\}, \\
& U_{2}=\{\zeta \in C: 0<\mathfrak{R}(\zeta) \leq-\mathfrak{I}(\zeta)\}
\end{aligned}
$$

and let $U_{J}^{*}=U_{j} \cap C^{*}(j=1,2)$. To begin with, we will show that (1.23) for $\xi \in U_{1}^{*}$ and $\eta \in U_{2}^{*}$ with $\xi \neq \eta$. By the strong Markov property, we obtain that for $\xi \in C^{*} \backslash L$ and $\eta \in C^{*} \backslash L$ with $\eta_{2}<0$,

$$
G_{L}(\xi, \eta)=G(\xi, \eta)-\sum_{j=1}^{\infty} H_{L}(\xi,-j) G(-j, \eta),
$$

where $G(\xi, \eta)=a(\xi-\tilde{\eta})-a(\xi-\eta)$ and $\tilde{\eta}=-\eta_{1}+i \eta_{2}$. The strong Markov property also gives the following Brownian analogue of (6.1)

$$
g_{L}(\xi, \eta)=g(\xi, \eta)-\int_{0}^{\infty} h_{L}(\xi,-y) g(-y, \eta) d y
$$

where $g(\xi, \eta)=(1 / \pi) \log (|\xi-\tilde{\eta}| /|\xi-\eta|)$. The term $C|\xi-\eta|^{-2}$ appearing in the right-hand side of (1.23) comes from the difference between $G(\xi, \eta)$ and $2 g(\xi, \eta)$ (see (1.6)). It remains to show that for $\xi \in U_{1}^{*}$ and $\eta \in U_{2}^{*}$ with $\xi \neq \eta$,

$$
\left|\sum_{j=1}^{\infty} H_{L}(\xi,-j) G(-j, \eta)-2 \int_{0}^{\infty} h_{L}(\xi,-y) g(-y, \eta) d y\right| \leq C|\xi|^{-2 / 3}|\eta|^{-2 / 3}
$$

Recall the proof of (3.17). We rewrite the difference between the second term on the right-hand side of (6.1) and that of (6.2) with $h_{1}$ and $h_{2}$. (Take $H_{L}, G, h_{L}, 2 g$ for $H_{B}, H_{L}, h_{B}, h_{L}$, respectively.) Further, we decompose $h_{1}$ into two parts and $h_{2}$ into four parts (see (3.18), (3.22) and (3.23)). Then we can show that each term appearing in those decomposition is bounded in absolute value by a constant multiple of $|\xi|^{-2 / 3} l^{-2 / 3}$. Thus we have (6.3). 
If $\xi \in\left(C^{*} \backslash L\right) \backslash U_{1}^{*}$ and $\eta \in U_{2}^{*}$, then we obtain the relations

$$
G_{L}(\xi, \eta)=\sum_{j=1}^{\infty} H_{B}(\xi, j) G_{L}(j, \eta)
$$

and its Brownian analogue

$$
g_{L}(\xi, \eta)=\int_{0}^{\infty} h_{B}(\xi, y) g_{L}(y, \eta) d y
$$

Notice that $j \in U_{1}^{*}$ in (6.4), for which

$$
\left|G_{L}(j, \eta)-2 g_{L}(j, \eta)\right| \leq C j^{-2 / 3}|\eta|^{-2 / 3}
$$

as has already been shown above. Take $H_{B}, G_{L}, h_{B}, 2 g_{L}$ for $H_{B}, H_{L}, h_{B}, h_{L}$, respectively. The same procedure as in the proof of (3.17) verifies the estimate

$$
\left|\sum_{j=1}^{\infty} H_{B}(\xi, j) G_{L}(j, \eta)-2 \int_{0}^{\infty} h_{B}(\xi, y) g_{L}(y, \eta) d y\right| \leq C|\xi|^{-2 / 3}|\eta|^{-2 / 3}
$$

for $\xi \in\left(C^{*} \backslash L\right) \backslash U_{1}^{*}$ and $\eta \in U_{2}^{*}$ as desired with the help of (6.5).

Put

$$
\begin{aligned}
& U_{3}=\{\zeta \in C:-\mathfrak{I}(\zeta)<\mathfrak{R}(\zeta) \leq 0\}, \\
& U_{4}=\{\zeta \in C \backslash\{0\}: 0 \leq \mathfrak{R}(\zeta), 0 \leq \mathfrak{I}(\zeta)\}, \\
& U_{5}=\{\zeta \in C:-\mathfrak{R}(\zeta)<\mathfrak{I}(\zeta) \leq 0\},
\end{aligned}
$$

and let $U_{J}^{*}=U_{J} \cap C^{*}(j=3,4,5)$. We will show that (1.23) holds for $\xi, \eta \in U_{4}^{*}$ with $\xi \neq \eta$. In this case, the proof of $(1.23)$ is based on the relations

$$
G_{L}(\xi, \eta)=G_{B^{\prime}}(\xi, \eta)+\sum_{J \in Z \backslash\{0\}} H_{B^{\prime}}(\xi, j-i j) G_{L}(j-i j, \eta)
$$

and its Brownian analogue

$$
g_{L}(\xi, \eta)=g_{B^{\prime}}(\xi, \eta)+\int_{\boldsymbol{R} \backslash\{0\}} h_{B^{\prime}}(\xi, y-i y) g_{L}(y-i y, \eta) d y,
$$

where $B^{\prime}=\{\zeta \in C:-\mathfrak{R}(\zeta) \geq \mathfrak{I}(\zeta)\}$ as in Remark 1.2, $g_{B^{\prime}}$ is the Green function of $B^{\prime}$ for two-dimensional standard Brownian motion and explicitly given by $g_{B^{\prime}}(\xi, \eta)=(1 / \pi) \log (|\xi+i \bar{\eta}| /|\xi-\eta|)$. Combining (1.8) and (1.6), the term $C|\xi-\eta|^{-2}$ appearing in the right-hand side of (1.23) comes from the difference between $C_{B^{\prime}}(\xi, \eta)$ and $2 g_{B^{\prime}}(\xi, \eta)$. Since $G_{L}$ and $g_{L}$ are symmetric, i.e., $G_{L}(j-i j, \eta)=G_{L}(\eta, j-i j)=G_{L}(i \bar{\eta},-j+i j), g_{L}(j-i j, \eta)=g_{L}(\eta, j-i j)=$ $g_{L}(i \bar{\eta},-j+i j)$, the estimate $(1.23)$ for $\xi \in C^{*} \backslash L$ and $\eta \in U_{2}^{*}$ with $\xi \neq \eta$, as has already been shown above implies that $j \in Z \backslash\{0\}$ and $\eta \in U_{4}^{*}$,

$$
\left|G_{L}(j-i j, \eta)-2 g_{L}(j-i j, \eta)\right| \leq C|j|^{-2 / 3}|\eta|^{-2 / 3} .
$$




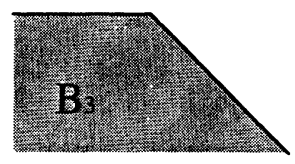

Figure 2: The region $B_{3}$.

Take $H_{B^{\prime}}, G_{L}, h_{B^{\prime}}, 2 g_{L}$ for $H_{B}, H_{L}, h_{B}, h_{L}$, respectively. The same procedure as in the proof of (3.17) also verifies the estimate

$$
\begin{aligned}
& \left|\sum_{j \in \boldsymbol{Z} \backslash\{0\}} H_{B^{\prime}}(\xi, j-i j) G_{L}(j-i j, \eta)-2 \int_{\boldsymbol{R} \backslash\{0\}} h_{B^{\prime}}(\xi, y-i y) g_{L}(y-i y, \eta) d y\right| \\
& \quad \leq C|\xi|^{-2 / 3}|\eta|^{-2 / 3}
\end{aligned}
$$

for $\xi, \eta \in U_{4}^{*}$ with $\xi \neq \eta$.

We will show that $(1.23)$ for $\xi \in U_{3}^{*} \cap U_{4}^{*}$ and $\eta \in U_{3}^{*}$ with $\xi \neq \eta$. In this case, the proof of (1.23) is based on the relations

$$
G_{L}(\xi, \eta)=G_{B_{3}}(\xi, \eta)+\sum_{j=1}^{\infty} H_{B_{3}}(\xi, j-i j) G_{L}(j-i j, \eta)
$$

and

$$
g_{L}(\xi, \eta)=g_{B_{3}}(\xi, \eta)+\int_{0}^{\infty} h_{B_{3}}(\xi, y-i y) g_{L}(y-i y, \eta) d y
$$

where $B_{3}=L \cup\{\zeta \in C: 0<\mathfrak{R}(\zeta) \leq-\mathfrak{I}(\zeta)\} \quad$ (see Figure 2) and $h_{B_{3}}(\xi, \cdot)$ and $g_{B_{3}}(\xi, \cdot)$ are the distribution and the Green function to $B_{3}$ of two-dimensional standard Brownian motion starting at $\xi$, respectively. To make further progress on the difference $G_{L}(\xi, \eta)-2 g_{L}(\xi, \eta)$, we will check that for $\xi \in C^{*} \backslash B_{3}$ and $\eta \in \partial\left(C^{*} \backslash B_{3}\right)$

$$
\left|H_{B_{3}}(\xi, \eta)-h_{B_{3}}(\xi, \eta)\right| \leq C\left\{|\xi-\eta|^{-3}+|\xi|^{-4 / 5}(|\eta|+1)^{-9 / 5}\right\}
$$

and for $\xi \in C^{*} \backslash B_{3}$ and $y \geq 1 / 2$,

$$
\begin{aligned}
& \left|H_{B_{3}}(\xi,\langle y\rangle-i\langle y\rangle)-h_{B_{3}}(\xi, y-i y)\right| \\
& \quad \leq C\left\{|\xi-y+i y|^{-2}+|\xi|^{-1 / 5} y^{-6 / 5}\left(|\xi|^{-3 / 5} \wedge y^{-3 / 5}\right)\right\} .
\end{aligned}
$$

Moreover, we will check that for $\xi \in U_{3}^{*} \cup U_{4}^{*}$ and $\eta \in U_{3}^{*}$ with $\xi \neq \eta$,

$$
\left|G_{B_{3}}(\xi, \eta)-2 g_{B_{3}}(\xi, \eta)\right| \leq C\left\{|\xi-\eta|^{-2}+|\xi|^{-4 / 5}|\eta|^{-4 / 5}\right\}
$$

and for $j \in N$ and $\eta \in U_{3}^{*}$,

$$
\left|G_{L}(j-i j, \eta)-2 g_{L}(j-i j, \eta)\right| \leq C j^{-2 / 3}|\eta|^{-2 / 3} .
$$


Then same procedure as in the proof of (3.17) implies that for $\xi \in U_{3}^{*} \cup U_{4}^{*}$ and $\eta \in U_{3}^{*}$ with $\xi \neq \eta$,

$$
\begin{aligned}
& \left|\sum_{J=1}^{\infty} H_{B_{3}}(\xi, j-i j) G_{L}(j-i j, \eta)-2 \int_{0}^{\infty} h_{B_{3}}(\xi, y-i y) g_{L}(y-i y, \eta) d y\right| \\
& \quad \leq C|\xi|^{-2 / 3}|\eta|^{-2 / 3}
\end{aligned}
$$

(taking $H_{B_{3}}, G_{L}, h_{B_{3}}, 2 g_{L}$ for $H_{B}, H_{L}, h_{B}, h_{L}$, respectively) and we conclude that (1.23) holds for $\xi \in\left(U_{3}^{*} \cup U_{4}^{*}\right)$ and $\eta \in U_{3}^{*}$ with $\xi \neq \eta$. It remains to check (6.6), (6.7), (6.8) and (6.9).

(6.9) is immediate from symmetry of $G_{L}$ and $g_{L}$ and (1.23) for $\xi \in C^{*} \backslash L$ and $\eta \in U_{2}^{*}$ with $\xi \neq \eta$. Let

$$
\hat{V}=\{\zeta \in C: \mathfrak{R}(\zeta) \leq \mathfrak{I}(\zeta)\} \cup\{\zeta \in C:-\mathfrak{R}(\zeta) \geq \mathfrak{I}(\zeta)\}
$$

and let

$$
\begin{aligned}
& \left(H_{B} \cdot H_{\hat{V}}\right)^{1}(k, l)=\sum_{j=1}^{\infty} H_{B}(k+i k, j) H_{\hat{V}}(j, l-i l) \\
& \left(H_{B} \cdot H_{\hat{V}}\right)^{n}(k, l)=\sum_{j=1}^{\infty}\left(H_{B} \cdot H_{\hat{V}}\right)^{n-1}(k, j)\left(H_{B} \cdot H_{\hat{V}}\right)^{1}(j, l) .
\end{aligned}
$$

We employ the relation

$$
H_{B_{3}}(k+i k, l-i l)=\sum_{n=1}^{\infty}\left(H_{B} \cdot H_{\hat{V}}\right)^{n}(k, l)
$$

instead of (2.4). In view of this relation, the method developed in $\$ 3$ can be adapted for deriving (6.6) and (6.7). The proof of (6.8) is similar to that of (1.23) for $\xi \in U_{1}^{*}$ and $\eta \in U_{2}^{*}$ with $\xi \neq \eta$. (6.8) follows from the relations

$$
G_{B_{3}}(\xi, \eta)=a(\xi-\bar{\eta})-a(\xi-\eta)-\sum_{j=1}^{\infty} H_{B_{3}}(\xi, j-i j)\{a(j-i j-\bar{\eta})-a(j-\imath j-\eta)\}
$$

and

$$
g_{B_{3}}(\xi, \eta)=\frac{1}{\pi} \log \frac{|\xi-\bar{\eta}|}{|\xi-\eta|}-\int_{0}^{\infty} h_{B_{3}}(\xi, y-i y) \frac{1}{\pi} \log \frac{|y-i y-\bar{\eta}|}{|y-i y-\eta|} d y
$$

that are given by the strong Markov property.

If $\xi \in U_{5}^{*}$ and $\eta \in U_{3}^{*}$, then we obtain the relations

$$
G_{L}(\xi, \eta)=G_{L}(\xi, \tilde{\eta})+G(\xi, \eta)-\sum_{j=1}^{\infty} H_{L}(\xi,-j) G(-j, \eta)
$$

and its Brownian analogue 


$$
g_{L}(\xi, \eta)=g_{L}(\xi, \tilde{\eta})+g(\xi, \eta)-\int_{0}^{\infty} h_{L}(\xi,-y) g(-y, \eta) d y
$$

Notice that $i \bar{\xi} \in U_{3}^{*}$ and $\eta_{2}-i \eta_{1} \in U_{4}^{*}$ if $\xi \in U_{5}^{*}$ and $\eta \in U_{3}^{*}$ and that $G_{L}$ and $g_{L}$ are symmetric. The estimate

$$
\left|G_{L}(\xi, \tilde{\eta})-2 g_{L}(\xi, \tilde{\eta})\right| \leq C\left\{|\xi-\eta|^{-2}+|\xi|^{-2 / 3}|\eta|^{-2 / 3}\right\}
$$

follow from (1.23) for $\xi \in U_{3}^{*} \cup U_{4}^{*}$ and $\eta \in U_{3}^{*}$ with $\xi \neq \eta$, as has already been shown above and the inequality $|\xi-\eta| \leq 4\left|i \bar{\xi}-\eta_{2}+i \eta_{1}\right|$ (recall $\xi \in U_{5}^{*}$ and $\left.\eta \in U_{3}^{*}\right)$. In view of $(6.10)$ and (6.11), the same argument as showing (1.23) for $\xi \in U_{1}^{*}$ and $\eta \in U_{2}^{*}$ with $\xi \neq \eta$ gives

$$
\left|G_{L}(\xi, \eta)-G_{L}(\xi, \tilde{\eta})-2 g_{L}(\xi, \eta)+2 g_{L}(\xi, \tilde{\eta})\right| \leq C\left\{|\xi-\eta|^{-2}+|\xi|^{-2 / 3}|\eta|^{-2 / 3}\right\} .
$$

Thus we have (1.23) for $\xi \in U_{5}^{*}$ and $\eta \in U_{3}^{*}$.

By symmetry of $G_{L}$ and $g_{L}$, we conclude that (1.23) for $\xi, \eta \in C^{*} \backslash L$ with $\xi \neq \eta$. The proof of Theorem 1.8 is complete.

\section{Appendix}

For $\beta \in(0,2 \pi)$, put

$$
W=W^{\beta}:=\{\xi \in C: \beta \leq \theta \leq 2 \pi\},
$$

where $(|\xi|, \theta)$ is the polar coordinate of $\xi, 0<\theta \leq 2 \pi$ and let $h_{W}(\xi, \cdot)$ be the hitting distribution to $W$ of two-dimensional standard Brownian motion starting at $\xi$. We will show that for $\xi \in \boldsymbol{C} \backslash W$ and $\eta \in W$ with $\arg (\eta)=\beta, h_{W}(\xi, \eta)$ is expressed as

$$
\begin{aligned}
h_{W}(\xi, \eta)= & \frac{\pi}{\beta^{2}} \sum_{m=1}^{\infty}(-1)^{m+1} m\left(\frac{r_{0} r}{r_{0}^{2}+r^{2}}\right)^{\hat{v}_{m}} r^{-1} \\
& \cdot\left\{\frac{1}{\hat{v}_{m}}+\left(\frac{r_{0} r}{r_{0}^{2}+r^{2}}\right)^{2}+\sum_{n=2}^{\infty} c\left(\hat{v}_{m}, n\right)\left(\frac{r_{0} r}{r_{0}^{2}+r^{2}}\right)^{2 n}\right\} \\
& \cdot \sin \frac{m \pi \theta_{0}}{\beta},
\end{aligned}
$$

where $\left(r_{0}, \theta_{0}\right)$ and $(r, \beta)$ are the polar coordinates of $\xi$ and $\eta, \hat{v}_{m}=m \pi / \beta$ and $c(v, n)$ is the same function as in (2.10).

The two-dimensional standard Brownian motion $W(t)$ can be expressed as the skew product

$$
\left(r(t), \theta\left(\int_{0}^{t} r^{-2}(s) d s\right)\right),
$$

where $r(t)$ is the two-dimensional Bessel process and $\theta(t)$ is the projection modulo $2 \pi$ of 1-dimensional standard Brownian motion that is independent of $r(t)$. 
Let $0<\theta_{0}<\beta$ and $T:=\inf \{t \geq 0: \theta(t) \notin(0, \beta)\}$. By the Markov property,

$$
P_{\theta_{0}}\{\theta(T)=\beta, T>t\}=E_{\theta_{0}}\left[P_{\theta(t)}\{\theta(T)=\beta\}: T>t\right] .
$$

It is known in Ito and McKean [3, p. 31] that for $0<\theta_{0}<\beta$,

$$
P_{\theta_{0}}\{\theta(t) \in d \theta, T>t\}=\frac{2}{\beta} \sum_{m=1}^{\infty} \exp \left(-\frac{m^{2} \pi^{2} t}{2 \beta^{2}}\right)\left(\sin \frac{m \pi \theta_{0}}{\beta}\right)\left(\sin \frac{m \pi \theta}{\beta}\right) .
$$

Further, it is easy to see that for $0<\theta<\beta, P_{\theta}\{\theta(T)=\beta\}=\theta / \beta$. Combining above relations and using an easy calculation, we have

$$
P_{\theta_{0}}\{\theta(T)=\beta, T>t\}=2 \sum_{m=1}^{\infty} \frac{(-1)^{m+1}}{m \pi} \exp \left(-\frac{m^{2} \pi^{2} t}{2 \beta^{2}}\right) \sin \frac{m \pi \theta_{0}}{\beta} .
$$

We write

$$
h\left(t, r_{0}, r\right) 2 r d r:=E_{r_{0}}\left[\exp \left(-\frac{v^{2}}{2} \int_{0}^{t} r^{-2}(s) d s\right), r(t) \in d r\right] .
$$

Then

$$
h\left(t, r_{0}, r\right)=\frac{1}{2 t} \exp \left(-\frac{r_{0}^{2}+r^{2}}{2 t}\right) \tilde{I}_{v}\left(\frac{r_{0} r}{t}\right)
$$

where $\Gamma(s)$ is the gamma function and

$$
\tilde{I}_{v}(x):=\left(\frac{x}{2}\right)^{v} \sum_{n=0}^{\infty} \frac{(x / 2)^{2 n}}{n ! \Gamma(v+n+1)}
$$

(see Ito and McKean [3, p. 271]).

Let $\hat{T}:=\inf \left\{t \geq 0: \theta\left(\int_{0}^{t} r^{-2}(s) d s\right) \notin(0, \beta)\right\}$. Since $\theta(t)$ is independent of $r(t)$, we can compute the hitting distribution to $W$ by writing

$$
\begin{aligned}
P_{\left(r_{0}, \theta_{0}\right)} & \left\{\theta\left(\int_{0}^{\hat{T}} r^{-2}(s) d s\right)=\beta, r(\hat{T}) \in d r\right\} \\
= & \int_{0}^{\infty} E_{r_{0}}\left[-\frac{d}{d t} P_{\theta_{0}}\left\{\theta\left(\int_{0}^{\hat{T}} r^{-2}(s) d s\right)=\beta, \hat{T}>t\right\} r(t) \in d r\right] d t .
\end{aligned}
$$

(7.2) and (7.3) imply that the right-hand side of above is equal to

$$
\frac{\pi}{\beta^{2}} \sum_{m=1}^{\infty}(-1)^{m+1} m r^{-1}\left(\sin \frac{m \pi \theta_{0}}{\beta}\right) \int_{0}^{\infty} t^{-1} \tilde{I}_{\hat{v}_{m}}\left(\frac{r_{0} r}{t}\right) \exp \left(\frac{r_{0}^{2}+r^{2}}{2 t}\right) d t .
$$

Noticing that for $v>1$, the integral of $t^{-v} \exp \left(-\left(r_{0}^{2}+r^{2}\right) /(2 t)\right)$ over $(0, \infty)$ with respect to $t$ is equal to $\left(2 /\left(r_{0}^{2}+r^{2}\right)\right)^{v-1} \Gamma(v-1)$, we obtain an expression of the hitting distribution to $W$.

Put $\beta=3 \pi / 2$ and replace $\theta_{0}$ by $\theta+(\pi / 2)$ in the right-hand side of (7.1). Then we have $(2.10)$ in $\$ 2$. 
Let $g_{W}$ be the Green function of the sector $W$. Then we have

$$
\begin{aligned}
g_{W}(\xi, \eta)= & \int_{0}^{\infty}\left\{E_{\xi}\left[r(t) \in d r, \theta\left(\int_{0}^{t} r^{-2}(s) d s\right) \in d \theta, T>\int_{0}^{t} r^{-2}(s) d s\right] / r\right\} d t \\
= & \frac{2}{\beta} \sum_{m=1}^{\infty}\left(\sin \frac{m \pi \theta_{0}}{\beta}\right)\left(\sin \frac{m \pi \theta}{\beta}\right) \\
& \cdot \int_{0}^{\infty} t^{-1} \tilde{I}_{\hat{v}_{m}}\left(\frac{r_{0} r}{t}\right) \exp \left(\frac{r_{0}^{2}+r^{2}}{2 t}\right) d t \\
= & \frac{2}{\beta} \sum_{m=1}^{\infty}\left(\sin \frac{m \pi \theta_{0}}{\beta}\right)\left(\sin \frac{m \pi \theta}{\beta}\right)\left(\frac{r_{0} r}{r_{0}^{2}+r^{2}}\right)^{\hat{v}_{m}} \\
& \cdot\left\{\frac{1}{\hat{v}_{m}}+\left(\frac{r_{0} r}{r_{0}^{2}+r^{2}}\right)^{2}+\sum_{n=2}^{\infty} c\left(\hat{v}_{m}, n\right)\left(\frac{r_{0} r}{r_{0}^{2}+r^{2}}\right)^{2 n}\right\},
\end{aligned}
$$

where $\left(r_{0}, \theta_{0}\right)$ and $(r, \theta)$ are the polar coordinates of $\xi$ and $\eta$.

Acknowledgement. I would like to express my sincere gratitude to Professor Kôhei Uchiyama of Tokyo Institute of Technology for valuable suggestions. I thank Professor Harry Kesten of Cornell University for sending the paper Kesten $[6]$.

\section{REFERENCES}

[1] AuER, P., Some hittıng probabilities of random walk on $Z^{2}$, Limit Theorems in Probability and Statıstıcs (L. Berkes, E. Cákı and P. Révész eds.), North Holland, 1990, 9-25.

[2] Fukai, Y AND UChiYama, K., Potential Kernel for two-dimensional random walk, Ann. Probab., 24 (1996), 1979-1992.

[ 3 ] Ito, K. And McKean, H. P., Diffusion Processes and their Sample Paths, Grundlehren Math. Wiss., 125, Springer-Verlag, 1974.

[4] Kesten, H., Hittıng probabilitıes of random walks on $Z^{d}$, Stochastıc Process. Appl., 25 (1987), 165-184.

[5] KESTEN, H., Relations between solutions to a discrete and contınuous Dirıchlet problem, Random Walks, Brownian Motıon and Interactıng Particle Systems (R. Durett and H. Kesten eds.), Birkhäuser, 1991, 309-321.

[6] Kesten, H., Relations between solutions to a discrete and contınuous Dirichlet problem II, preprint.

[ 7] LAWLER, G. F., Intersections of Random Walks, Birkhäuser, 1991.

[ 8 ] SpItzer, F., Prınciples of Random Walk, 2nd ed., Sprınger-Verlag, 1976.

Department of Applied Physics Tokyo Institute of TeChNology OH-OKayama, Meguro

TOKYO 152-8551

JAPAN

E-MAIL. fuka1@neptune.ap.titech.ac.jp
Graduate School of Mathematics KYUSHU UNIVERSITY FUKUOKA 812-8581 JAPAN 